This document is the Accepted Manuscript version of a Published Work that appeared in final form in Macromolecules 54(1) : 259-271 (2021), copyright (C) 2020 American Chemical Society after peer review and technical editing by the publisher. To access the final edited and published work see https://doi.org/10.1021/ acs.macromol.0c02374

\title{
Even-odd Effect in Aliphatic Polycarbonates with Different Chain Lengths: from Poly (hexamethylene carbonate) to Poly (dodecamethylene carbonate)
}

\author{
Ricardo A. Pérez-Camargo ${ }^{1}$, Leire Meabe ${ }^{2}$, Guoming Liu ${ }^{1,3 *}$, Haritz Sardon ${ }^{2}$, Ying \\ Zhao $^{1,3}$, Dujin Wang ${ }^{1,3}$, Alejandro J. Müller ${ }^{2,4^{*}}$
}

${ }^{1}$ Beijing National Laboratory for Molecular Sciences, CAS Key Laboratory of Engineering Plastics, CAS Research/Education Center for Excellence in Molecular Sciences, Institute of Chemistry, Chinese Academy of Sciences, Beijing 100190, China

\footnotetext{
${ }^{2}$ POLYMAT and Faculty of Chemistry, University of the Basque Country UPV/EHU, Paseo Manuel de Lardizabal 3, 20018, Donostia-San Sebastián, Spain.

${ }^{3}$ University of Chinese Academy of Sciences, Beijing 100049, China

${ }^{4}$ IKERBASQUE, Basque Foundation for Science, Bilbao, Spain.
}

*Corresponding authors: gmliu@iccas.ac.cn and alejandrojesus.muller@ehu.es 
For Table of Content use only

\section{Even-odd Effect in Aliphatic Polycarbonates with Different Chain Lengths: from Poly (hexamethylene carbonate) to Poly (dodecamethylene carbonate)}

Ricardo A. Pérez-Camargo ${ }^{1}$, Leire Meabe ${ }^{2}$, Guoming Liu ${ }^{1,3 *}$, Haritz Sardon $^{2}$, Ying Zhao $^{1,3}$, Dujin Wang ${ }^{1,3}$, Alejandro J. Müller ${ }^{2,4^{*}}$

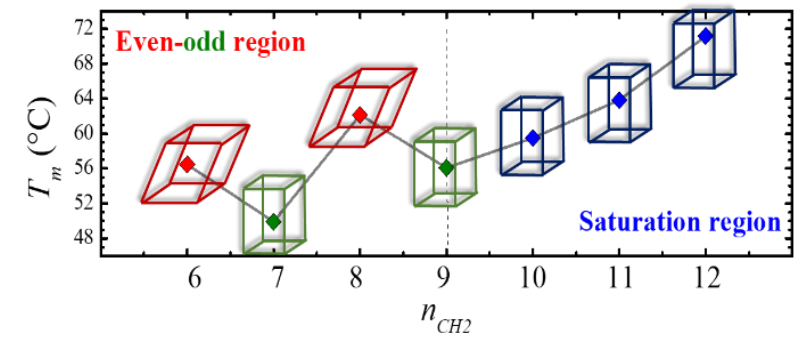




\begin{abstract}
We have characterized a series of aliphatic polycarbonates synthesized by organocatalysis containing a variable number of methylene groups $\left(n_{\mathrm{CH} 2}\right)$ in their repeat units ranging from $n_{\mathrm{CH} 2}=6$ to 12 . The melting and crystallization behavior and crystalline structures were studied by differential scanning calorimetry (DSC), Fourier transform infrared spectroscopy (FT-IR), and wide-angle X-ray scattering (WAXS). We found a clear even-odd effect in terms of thermal properties and crystalline structure, for $n_{\mathrm{CH} 2}=6$ to 9 , and a saturation of the even-odd effect, for $n_{\mathrm{CH} 2}=10$ to 12 . These results were independent of the crystallization conditions employed: nonisothermal, isothermal and successive self-nucleation and annealing (SSA). The evenodd region showed that the even samples had higher melting temperatures than the odd ones, and a monoclinic unit cell. On the other hand, the odd samples showed an orthorhombic unit cell. Both even and odd samples exhibited a trans-conformation, with a dilution of the impact of carbonyl group as evidenced by the weakening of the crystalline memory effect as $n_{\mathrm{CH} 2}$ increases, independently of the even or odd nature of the samples. In the saturation region, the methylene instead of the carbonyl groups dominated the behavior, resulting in thermal properties that changed almost linearly with $n_{C H 2}$. The unit cells were all orthorhombic and the strength of memory effect was similar, as $n_{\mathrm{CH} 2}$ increased. Accordingly, the samples showed a shift of the FTIR bands towards a PE-like dominated conformation.
\end{abstract}

Keywords: aliphatic polycarbonates, even-odd effect, saturation of even-odd effect, isothermal crystallization, Successive Self-Nucleation and Annealing. 


\section{INTRODUCTION}

Biodegradable polymers represent a solution to the pollution problems generated by plastics. Among them, the most important groups are the aliphatic polyesters and polycarbonates. ${ }^{1}$ Polycaprolactone, poly (lactic acid), poly (butylene succinate), poly (glycolic acid), among others, have suitable properties to meet the demands in agriculture, packaging, and medical applications. ${ }^{2}$ One growing group of biodegradable polymers is that of aliphatic polycarbonates (PC)s. They have excellent biocompatibility, nontoxicity, chemical and physical properties. ${ }^{1,3-6}$ The PCs can degrade by a surface erosion process without producing acid compounds. ${ }^{7-9}$ This unique degradation process is suitable for environmental conservation, biomedicine, and pharmaceutics (e.g., in vivo medical application ${ }^{10,11}$ ). Because of the much lower degradation rate in comparison with polyesters, PCs are suitable for applications that require higher stability. Also, they have other interesting characteristics, such as high ionic conductivity, good electrochemical stability, and high lithium transfer number, which make them excellent candidates for solid polymer electrolytes. ${ }^{12}$

Researchers have used different synthetic routes to obtain PCs, including copolymerization of dioxides with epoxides, ring-opening polymerization (ROP), and polycondensation. ${ }^{3}{ }^{12}$ The copolymerization of dioxides with epoxides, despite leading to molecular weights (MW) as high as $300 \mathrm{~kg} / \mathrm{mol}$, in most of the cases also produce ether linkages within the chains that impair the thermal and mechanical properties. ${ }^{3}$ The chemical structure limits this method since it only allows to synthesize PCs from three- and four-membered cyclic epoxides. ${ }^{3,12}$ The ROP also allows obtaining PCs of high MW, but the availability of cyclic monomers 
undergoing ROP limits the chemical structure of the PCs. ${ }^{12}$

In the case of the polycondensation, different strategies have been reported. Initially, the polycondensation of aliphatic diols with phosgene was the most studied but due to environmental concerns in the last decade other reagents have been explored to replace phosgene and derivaties. ${ }^{3}$ Thus, the polycondensation of dialkyl carbonate and diols in the melt was developed to overcome this problem. However, this kind of polycondensation is usually catalyzed by metals, including $\mathrm{TiO}_{2} / \mathrm{SiO}_{2}{ }^{3}$ TSP-44, ${ }^{13}$ and sodium acetylacetonate. ${ }^{14}$ Even though they provide PCs with high $\mathrm{MW},{ }^{15}$ they remain as potential toxic impurities, which are not desired for biomedical applications.

The described situation has been improved with organocatalysts. ${ }^{16,17}$ Among organocatalysts, 4-dimethylaminopyridine (DMAP) shows the best catalytic performance ${ }^{18,19}$ and allows obtaining different PCs, ${ }^{12,18}$ and copolycarbonates. ${ }^{20}$ Despite the production of different PCs (i.e., with different content of methylene groups, $n_{C H 2}$, in the polymer repeating units), previous studies have been focused on the degradation properties, ${ }^{6}$ applications, ${ }^{12}$ and only a few works on the structure $(e . g$. , "Brill transition" in poly (octamethylene carbonate) ${ }^{13}$ ), and the memory effect. ${ }^{21}$ However, the crystallization behavior has not been explored in detail, leaving interesting phenomena, such as the even-odd effect, pending of investigation.

The even-odd effect consists of the alternation of the physical properties of the solid-state, such as melting points, sublimation, enthalpies, solubility, and modulus as a function of the number (even or odd) of methylene groups $\left(n_{\mathrm{CH} 2}\right)$ within the repeating units of the polymer under consideration. ${ }^{22}$ Although this effect has been studied in the last century, it is only since the last decade that its molecular origin has 
been studied in more detail, especially in those materials in which it is more pronounced: (a) thermotropic liquid crystalline phases, (b) $n$-alkanes and some $n$-alkyl derivatives at a relatively low number of $\mathrm{CH}_{2}$ groups and (c) in the presence of functional groups, in both chain extremities in comparison with monosubstituted alkanes. $^{23}$ Also, there are studies in aliphatic polyesters, polyamides, and polyimides, ${ }^{24-26}$ but only two studies are directly or indirectly related to the even-odd effect in aliphatic polycarbonates. ${ }^{1,12}$

Su et al. ${ }^{1}$ studied a series of PCs, $n_{C H 2}=3,4,5,6$, and 10, obtained by ROP. They found that the odd sample $\left(n_{\mathrm{CH} 2}=5\right)$ crystallized slower than the even ones $\left(n_{C H 2}=4,6\right.$, and 10), and attributed this behavior to an even-odd effect. This effect was also reflected in the melting temperature $\left(T_{\mathrm{m}}\right)$ fluctuation as $n_{\mathrm{CH} 2}$ increased, and a different WAXS pattern of the odd sample $\left(n_{\mathrm{CH} 2}=5\right)$ was obtained in comparison with those of the even samples $\left(n_{\mathrm{CH} 2}=4\right.$ and 10). The chemical structure and reduced symmetry, caused by pentylene units of the PC with $n_{\mathrm{CH} 2}=5$, explained the differences. Similar arguments were used to describe the absence of crystallization in the PC with $n_{\mathrm{CH} 2}=3$. Even though Meabe et al. ${ }^{12}$ focused on using PCs as solid polymer electrolytes, they also found $T_{m}$ fluctuations in PC with $n_{C H 2}=4,6$ to 10 , and 12, attributing them to an even-odd effect. Despite the advancements provided by $\mathrm{Su}$ et al., ${ }^{1}$ and Meabe et al. ${ }^{12}$ a clear understanding of the even-odd effect is still lacking. The reasons for this are: (a) only one odd sample was useful (the PC with $n_{C H 2}=3$ did not crystallize), (b) the absence of a continuous range of samples (ROP did not allow obtaining samples with $n_{\mathrm{CH} 2}=7$ to 9 ), and (c) the studies on the even and odd samples were not focused on the even-odd effect.

As far as the authors are aware, the study of Su et al. ${ }^{1}$ and Meabe et al. ${ }^{12}$ are 
the only ones related to the even-odd effect on polycarbonates. In this work, we study a wider range of PCs with different chain lengths, $n_{\mathrm{CH} 2}=6,7,8,9,10,11$, and 12 , including several odd samples $\left(n_{C H 2}=7,8,9\right.$ and 11) for better comparisons, through a systematic characterization of the crystallization behavior, structure, and molecular conformation, to better understand the even-odd effect in PCs.

\section{EXPERIMENTAL}

\subsection{Materials}

The aliphatic polycarbonates (PCs) were synthesized, following the procedure reported in a previous work, ${ }^{12}$ by conventional polycondensation of aliphatic diols (e.g., 1,10-decanediol; 1,12-dodecanediol) and dimethyl carbonate, using an organocatalyst (4-dimethilaminopyridine, DMAP) under melt-condensation conditions at 130 and $180{ }^{\circ} \mathrm{C}$. More details of the synthesis can be found in References 12 and 18 .

The described method allows obtaining a wide range of PCs with a variable number of methylene groups $\left(n_{\mathrm{CH} 2}\right)$ in between the carbonate group. Thus, $n_{\mathrm{CH} 2}$ values in the range 6 to 12 were obtained, with the molecular weights, dispersity indexes, and glass transition temperatures indicated in Table 1 . The general chemical structure of the aliphatic polycarbonate obtained is shown in Scheme 1.

Table 1. Number average molecular weight $\left(M_{n}\right)$ and dispersity index $(\nexists)$ of the obtained aliphatic polycarbonates. For details of the chemical characterization see our previous work in Reference 12.

\begin{tabular}{ccccc}
\hline $\boldsymbol{P C s}$ & $\boldsymbol{n}_{\boldsymbol{C H} \mathbf{2}}$ & $\boldsymbol{M}_{\boldsymbol{n}}(\mathrm{g} / \mathrm{mol})$ & $\boldsymbol{D}$ & $\boldsymbol{T}_{\boldsymbol{g}}\left({ }^{\circ} \mathrm{C}\right)$ \\
\hline PC6 & 6 & 25100 & 1.65 & -50 \\
PC7 & 7 & 14800 & 1.46 & -50 \\
\hline
\end{tabular}




\begin{tabular}{ccccc}
\hline PC8 & 8 & 15300 & 1.38 & -54 \\
PC9 & 9 & 16100 & 1.53 & -48 \\
PC10 & 10 & 8000 & 1.53 & -37 \\
PC11 & 11 & 8000 & 1.56 & -37 \\
PC12 & 12 & 8100 & 1.63 & -37 \\
\hline
\end{tabular}

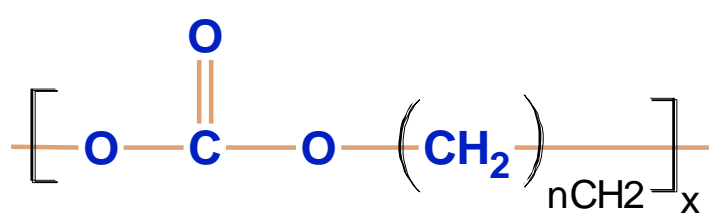

Scheme 1. General chemical structure of the aliphatic polycarbonate, $n_{\mathrm{CH} 2}$ represents the number of methylene units between carbonate groups, and $x$ the degree of polymerization. In this work, $n_{\mathrm{CH} 2}$ was systematically varied between 6 and 12 .

\subsection{Differential Scanning Calorimetry (DSC) experiments}

The DSC experiments were performed in a Perkin Elmer DSC 8500, connected to a controlled liquid nitrogen accessory (CLN2). The DSC was operated with a constant flow $(20 \mathrm{~mL} / \mathrm{min})$ of ultrapure nitrogen. It was calibrated with indium and zinc standards, and a sample weight of circa. $5 \mathrm{mg}$ was used. The cooling and heating scans were performed at $20{ }^{\circ} \mathrm{C} / \mathrm{min}$, except for isothermal experiments (i.e., cooling rate of $60{ }^{\circ} \mathrm{C} / \mathrm{min}$ ). The different employed protocols are described below:

\section{Non-isothermal test}

The thermal response during non-isothermal conditions was obtained as follows: the samples were (a) heated from room temperature until $T$ (i.e., temperature for erasing the thermal history) at $20{ }^{\circ} \mathrm{C} / \mathrm{min}$, recording the first heating, and then held during 3 minutes at $T$ to erase the thermal history. (b) The sample was cooled from $T$ 
to $-40{ }^{\circ} \mathrm{C}$ at $20{ }^{\circ} \mathrm{C} / \mathrm{min}$, registering the cooling scan, and held at $-40{ }^{\circ} \mathrm{C}$ for 1 minute. Finally, the (c) second heating scan was recorded from -40 to $T$ at $20{ }^{\circ} \mathrm{C} / \mathrm{min}$. Ratedependent experiments were also performed by changing the cooling rate (i.e., 5, 10, and $50{ }^{\circ} \mathrm{C} / \mathrm{min}$ ) in step (b).

\section{Isothermal test and modified isothermal test}

We perform the isothermal test by following the protocol of Lorenzo et al. ${ }^{27}$ Thus, we first determined the minimum crystallization temperature $\left(T_{c, \min }\right)$, and used it as the starting point of the isothermal experiments.

In the isothermal experiments, the sample was cooled from the melt until $T_{c}$ $\left(T_{c} \geq T_{c, \min }\right)$ at $60{ }^{\circ} \mathrm{C} / \mathrm{min}$, and held at $T_{c}$ enough time to reach the complete crystallization. In the above step, we register the isothermal crystallization behavior of the sample. Then, the sample was heated from $T_{c}$ to record its subsequent melting. This procedure was performed with at least 10 different crystallization temperatures.

One modification of the isothermal test was also performed. This consists in self-nucleating the sample, at the ideal self-nucleation temperature (see details below), before the isothermal step. With such procedure, the sample is ideally self-nucleated before the isothermal step. Therefore, when the isothermal crystallization is recorded, only the growth is recorded instead of the overall response (nucleation + growth). The details are explained in Scheme S1 (Section S1 of the SI).

Self-nucleation (SN), and Self-nucleation and Successive Annealing (SSA)

\section{experiments}

We performed SN experiments to all the samples, to determine their SN domains (Domain I, IIa, IIb, and III), and their ideal self-nucleation temperatures $\left(T_{s,}\right.$ 
ideal). We used a scan rate of $20{ }^{\circ} \mathrm{C} / \mathrm{min}$. The schematic representation of the thermal protocol is shown in Scheme S2 (Section S2 of the SI).

After determining the $T_{s \text {, ideal }}$ of each material, we performed SSA experiments according to the protocols established and reviewed by Müller et al. ${ }^{28-31}$ See more details in Scheme S3 (Section S2 of the SI).

We used cooling and heating rates of $20{ }^{\circ} \mathrm{C} / \mathrm{min}$, holding times of 5 minutes, and fractionation windows of $5{ }^{\circ} \mathrm{C}$. The SSA experiments (see Scheme S3) were performed with two approaches: starting with (a) the $T_{s, \text { ideal }}$ of each material, and (b) the highest $T_{s, \text { ideal }}$, corresponding to $68{ }^{\circ} \mathrm{C}$ (i.e., PC12). We used the latter approach for comparison purposes.

\subsection{X-ray characterization: Wide-angle X-ray scattering}

The X-ray scattering experiments were performed on a Xeuss 2.0 system (Xenocs SA), equipped with a microfocus CuKa X-ray source (GeniX3D, 50 kV, 0.6 mA). The detector used was a Pilatus 300K (DECTRIS, Swiss). The sample-todetector distance was $189.6 \mathrm{~mm}$, and the exposure time 300 seconds.

The sample was placed in the hot stage of a Linkam THMS600 connected to a liquid nitrogen station and the non-isothermal test employed during the DSC experiments was reproduced. The WAXS patterns were taken at different temperatures: at room temperature, in the molten state (i.e., at $T=$ temperature of erasing thermal history), at $-40{ }^{\circ} \mathrm{C}$, at the thermal transitions (e.g., crystallization and melting temperatures), and $3{ }^{\circ} \mathrm{C}$ below and above them.

\subsection{FT-IR Characterization}

We used a Nicolet 6700 FT-IR spectrometer of Thermo Fisher, connected to a 
Linkam FTIR 600 Hot Stage coupled to a Liquid Nitrogen supply. All the samples were film-cast in chloroform (by dissolving $5 \mathrm{mg}$ of sample in $1 \mathrm{~mL}$ of $\mathrm{CHCl}_{3}$ ) onto potassium bromide $(\mathrm{KBr})$ plates. The thermal protocol described in Section 2.2 for the non-isothermal DSC test, was reproduced in the hot-stage. In this case, for taking the FT-IR spectra, we used holding times of 1 minute and resolution of 2.0 or $4.0 \mathrm{~cm}^{-1}$ at each selected temperature and coaddition of 32 scans per spectra. The FT-IR spectra were taken at $-40{ }^{\circ} \mathrm{C}$, at $T$, at the thermal transitions detected previously in the DSC, and $5{ }^{\circ} \mathrm{C}$ above and below them.

\section{RESULTS AND DISCUSSION}

\subsection{Non-isothermal DSC}

Figure 1 shows the DSC cooling (Figure 1a), and second heating (Figure 1b) scans for all the samples. All the samples show a single crystallization peak. In the case of the second heating scans shown in Figure $1 \mathrm{~b}$, some samples exhibit a single melting peak (PC11, PC10, PC8), while others have a more complex melting. PC9 and PC12 show broad melting peaks with high temperature shoulders; while PC6 and PC7 exhibit two clearly separated melting peaks (see Figure 1b). 

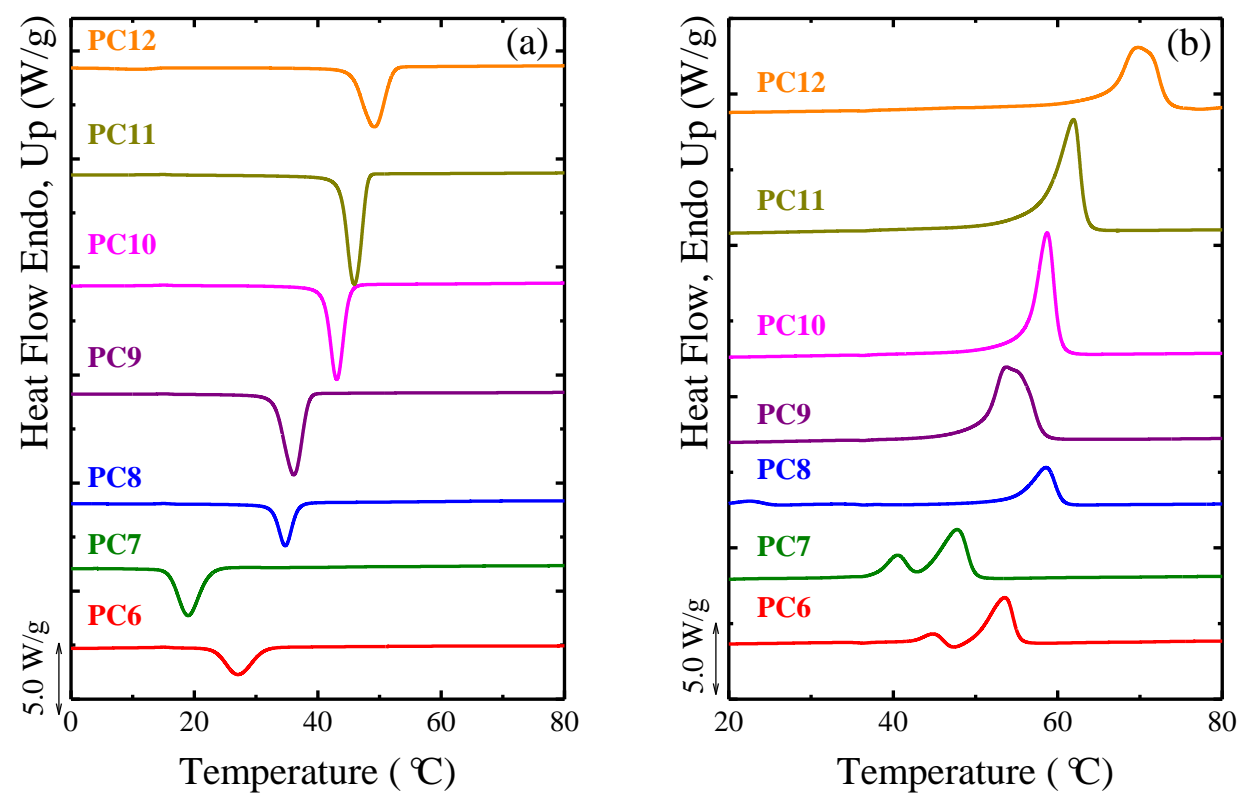

Figure 1. DSC (a) cooling, and (b) heating scans at $20{ }^{\circ} \mathrm{C} / \mathrm{min}$ for all the samples.

The double melting peaks in PC6 and PC7 are related to the reorganization process that is common in polyesters, as well as nylons and polyurethanes. ${ }^{32}$ Berti et al. ${ }^{33}$ attributed the complex melting behavior of the PC6 to a reorganization process during the scan. DSC scans at different cooling rates, as well as X-rays experiments, corroborates this behavior (see Figure S1).

The PC6 and PC8 samples also show exothermic and endothermic peaks below $25{ }^{\circ} \mathrm{C}$ (e.g., endothermic peaks at 6 and $20{ }^{\circ} \mathrm{C}$ for the PC6 and PC8, respectively). These transitions are not observed in Figure $1 \mathrm{~b}$ because of the scale employed. These peaks might be related to a solid-solid transition. Further details of this transition are going to be investigated in future work. 


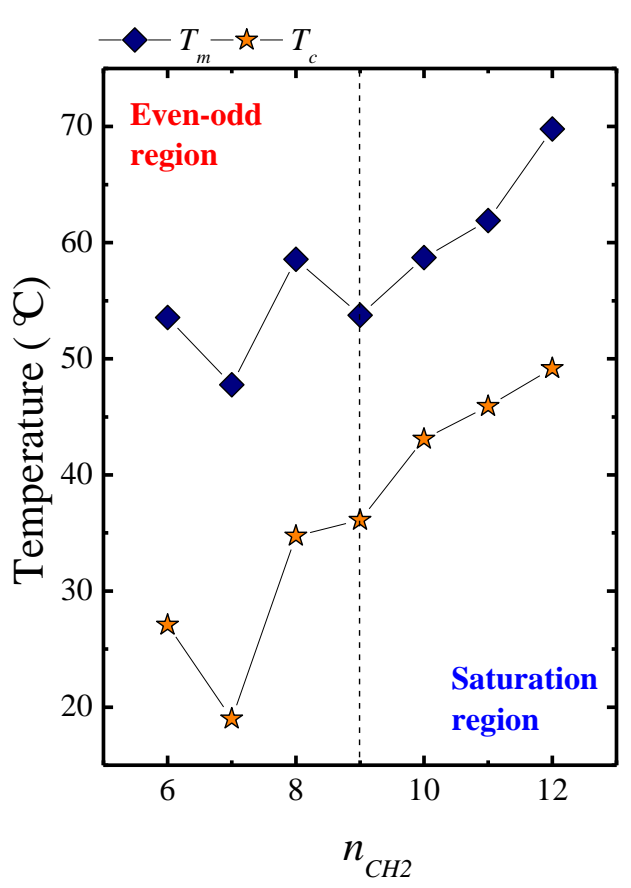

Figure 2. Crystallization and melting temperatures as a function of the number of methylene groups in the repeating unit of the polycarbonates employed $\left(n_{\mathrm{CH} 2}\right)$. Note that the highest melting temperature was taken for $n_{\mathrm{CH} 2}=6$ and 7 (see Figure 1b). The dashed line divides the plot in the even-odd effect region (left) and the saturation region (right).

Besides the different transitions, Figure 1 shows an alternate or zigzag trend (i.e., even samples have higher properties than odd ones) of both crystallization $\left(T_{c}\right)$ and melting $\left(T_{m}\right)$ peaks as the number of methylene groups in the repeating unit of the polycarbonate, $n_{\mathrm{CH} 2}$, increases up to 9 . Then, both $T_{c}$ and $T_{m}$ increase linearly as $n_{\mathrm{CH} 2}$ increases, for $n_{C H 2}=10$ to 12 . For the sake of clarity, we have plotted in Figure 2, the thermal transitions as a function $n_{C H 2}$. The thermal transitions are listed in Table S3.

Figure 2 can be divided into two regions, the even-odd region, $n_{\mathrm{CH} 2}=6$ to 9 , and the saturation region, $n_{\mathrm{CH}_{2}}=10$ to 12 . The even-odd region clearly shows an alternation of $T_{c}$ and $T_{m}$ values as $n_{C H 2}$ increases, due to an even-odd effect. In this case, even members show higher $T_{c}$ and $T_{m}$ than the odd ones. The observed even-odd 
effect is related to the inter and intramolecular interactions between structural groups, in this case, the $\mathrm{C}=\mathrm{O}$ groups. Such interactions are used to theoretically predict an alternated or zigzag behavior of the equilibrium melting point, up to $n_{\mathrm{CH} 2}=20$, by using the molar melt transition function derived by Van Krevelen ${ }^{34}$ (see Figure S2). In this work, we have found that the even-odd effect is originated by structural and conformational differences between the even and odd samples that will be discussed in Sections 3.4 and 3.5.

In this work, we found a saturation of the even-odd effect at $n_{\mathrm{CH} 2}>9$. Such saturation is reflected in the linear relationship of $T_{c}$ and $T_{m}$ as $n_{C H 2}$ increases (see Figure 2). Also, in Section 3.4 we show that in the saturation region, the crystalline structure remains unchanged despite increasing $n_{\mathrm{CH} 2}$. To get more insights into the even-odd effect in PCs, we performed isothermal crystallization experiments.

\subsection{Isothermal DSC test}

The isothermal conditions are closer to the thermodynamic equilibrium than the non-isothermal ones. To get more insights of the even-odd effect in this state, we performed isothermal DSC tests to study only the growth (i.e., modified isothermal test) and the overall crystallization (nucleation and growth) of all the samples.

\subsubsection{Isothermal DSC: Growth only (self-nucleated samples)}

In this work, we have implemented a modified isothermal DSC test to first produce the nucleation step in the material (by performing self-nucleation at the ideal self-nucleation temperature) and therefore only follow isothermally the growth or secondary nucleation contribution by DSC, as Lorenzo and Müller ${ }^{35}$ demonstrated previously for polyethylene, poly ( $\varepsilon$-caprolactone) and poly (p-dioxanone). We have implemented this technique due to the limitations to follow the spherulitic growth of 
the analyzed aliphatic polycarbonates by polarized light optical microscopy, because of the high nucleation density present in all samples.
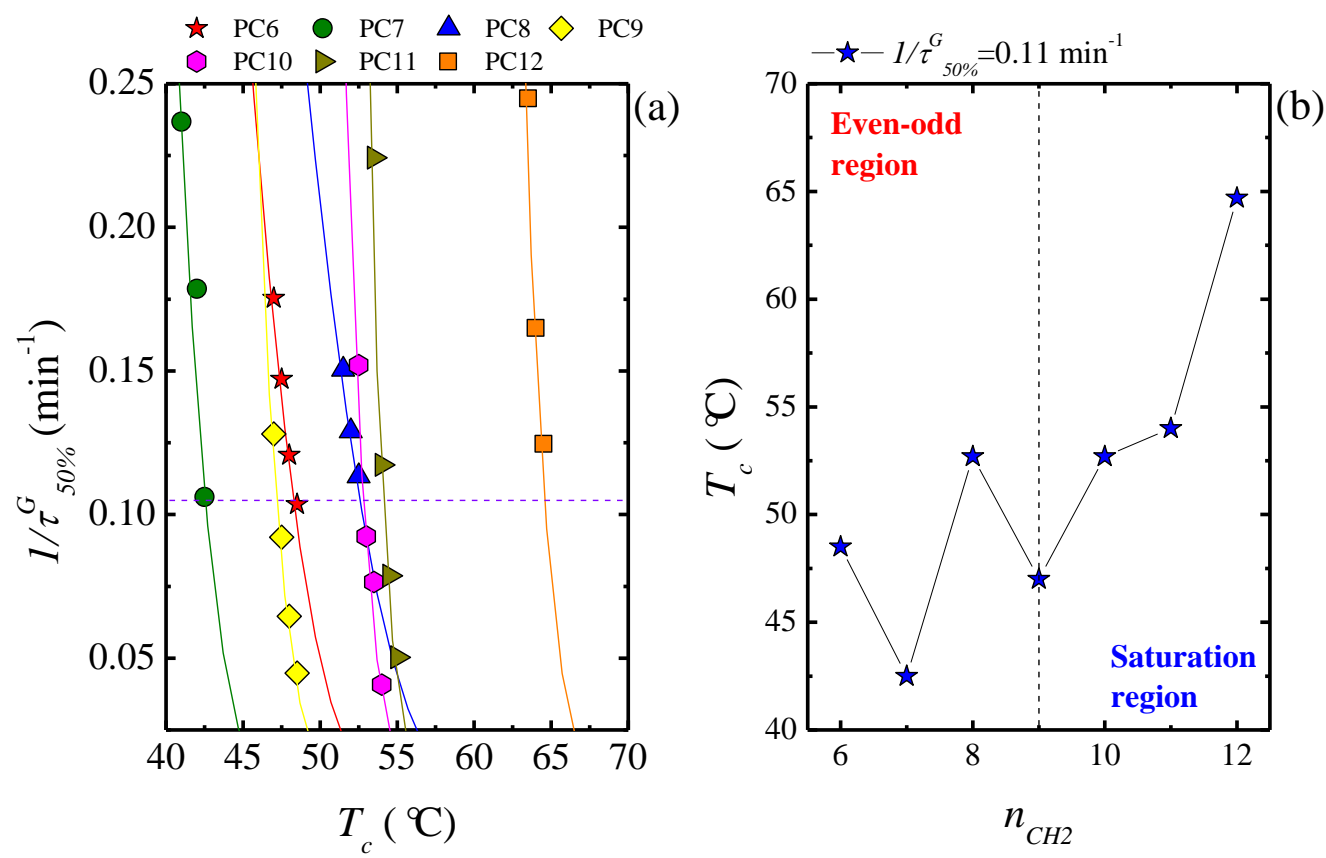

Figure 3. (a) The inverse of the crystallization half-time after ideally self-nucleating the material (i.e., $1 / \tau^{G}{ }_{50 \%}$ ) versus $T_{c}$; (b) $T_{c}$ versus $n_{C H 2}$ at a constant $1 / \tau^{G}{ }_{50 \%}=0.11 \mathrm{~min}^{-}$ 1. The solid lines in Figure 3a represent the Lauritzen and Hoffman fit to the data (see more details in Section S4 of the SI).

Figure $3 \mathrm{a}$ shows the quantity $1 / \tau^{G}{ }_{50 \%}$ that is proportional to the growth rate obtained by $\operatorname{DSC}^{35}$ as a function of $T_{c}$ for all the materials. In all cases, the growth rate is a decreasing function of $T_{c}$, as expected for flexible semi-crystalline polymers crystallizing at low supercoolings in the temperature range dominated by secondary nucleation. ${ }^{36}$ For comparison purposes, from Figure 3a, we have taken a constant rate, $1 / \tau^{G}{ }_{50 \%}=0.11 \mathrm{~min}^{-1}$, and plotted the corresponding $T_{c}$ values as a function of $n_{\mathrm{CH} 2}$ (see Figure 3b).

Figure 3 a shows that the $1 / \tau_{50 \%}^{G}$ vs. $T_{c}$ curves for $n_{C H 2}=6$ to 9 , alternate their positions. The even samples (i.e., $n_{\mathrm{CH} 2}=6$ and 8 ) crystallized at higher $T_{c}$ than the odd 
ones (i.e., $n_{\mathrm{CH} 2}=7$ and 9), responding to a strong even-odd effect. In contrast, the curves for $n_{\mathrm{CH} 2}=10$ to 12 , increases their $T_{c}$ as $n_{\mathrm{CH} 2}$ increases, due to an even-odd effect saturation. This behavior is in line with that found in the non-isothermal tests presented above (Figure 2).

The difference between the even-odd effect and the saturation of the even-odd effect can be observed in Figures 3b. As in the non-isothermal experiments, the plots can be divided into an even-odd region and a saturation region, demonstrating that these two behaviors also occur from the crystal growth point of view. In the next section, we present the overall (nucleation+growth) isothermal crystallization point of view.

\subsubsection{Isothermal DSC: Overall Crystallization}

Figure $4 \mathrm{a}$ shows the experimental values of the inverse of the half crystallization time $\left(1 / \tau_{50 \%}\right)$ that represent a measure of the overall crystallization rate (including both nucleation and growth) as a function of the isothermal crystallization temperature $\left(T_{c}\right)$. In all cases the crystallization rate decreases with $T_{c}$, as expected in the analysed temperature range. 


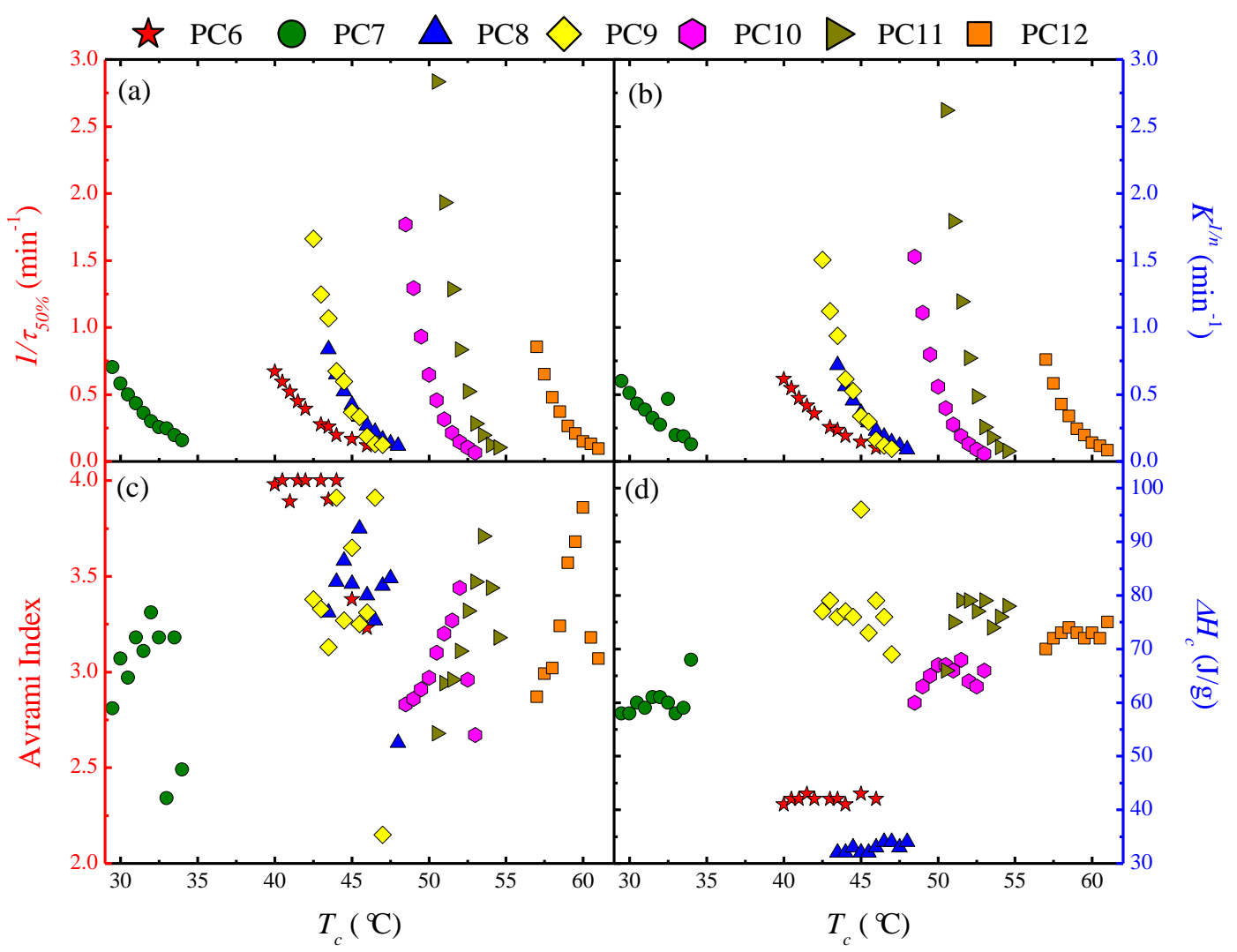

Figure 4. (a) Experimental overall half-crystallization rates $\left(1 / \tau_{50 \%}\right)$, (b) $K^{1 / n}$ values, (c) Avrami index, and (d) Enthalpies $\left(\Delta H_{c}\right)$ taking from isothermal curves, as a function of the isothermal crystallization temperatures $\left(T_{c}\right)$.

The crystallization rate versus $T_{c}$ curves in Figure 4 a show temperature ranges that do not follow a linear trend with $n_{\mathrm{CH} 2}$ for the PC6 to the PC9 samples, as expected for a strong even-odd effect. This region shows how the experimental data of the odd samples $\left(n_{\mathrm{CH} 2}=7\right.$, and 9$)$ are shifted to lower $T_{c}$ values with respect to the curves of the even samples $\left(n_{\mathrm{CH} 2}=6\right.$ and 8$)$. A similar behavior has also been observed in polyesters in a range of $n_{\mathrm{CH} 2}=2$ to 4 , in which the sample with $n_{\mathrm{CH} 2}=3$ crystallizes at the lowest $T_{c}$ range. ${ }^{25}$ Papageorgiou and Bikiaris attributed the faster crystallization of the poly (butylene succinate), $n_{\mathrm{CH} 2}=4$, to the flexible butylene units, and the retardation in the poly (propylene succinate), $n_{\mathrm{CH} 2}=3$, to its reduced symmetry caused by the propylene units. ${ }^{25}$ 
In the region of $n_{C H 2}=10$ to 12 (see the highest $T_{c}$ values in Figure $4 \mathrm{a}$ ), as $n_{\mathrm{CH} 2}$ increases, the $1 / \tau_{50 \%}$ vs. $T_{c}$ curves are constantly shifted to higher $T_{c}$, once again reflecting a saturation of the even-odd effect (as was observed during the nonisothermal and modified isothermal tests). Such saturation is the result of increasing the methylene segments that limits the interactions of the carbonate groups in the chains.

The isothermal DSC curves were analyzed with the Avrami theory ${ }^{37}$ (see details on Section S5 the SI), and the experimental overall crystallization rates $\left(1 / \tau_{50 \%}\right)$ as a function of $T_{c}$ with the Lauritzen and Hoffman theory ${ }^{38,39}$ (see Section $\mathrm{S} 4$ for more details).

The constant $K$ of the Avrami equation (see Section S5 of the SI) represents the overall crystallization rate constant with units that depend on the Avrami index $\left(e . g ., \min ^{-\mathrm{n}}\right)$. In order to compare different $K$ values with the same units, $K$ should be elevated to the power $1 / n$. Figure $4 \mathrm{~b}$ shows how $K^{1 / n}$ values, obtained after fitting the data with the Avrami theory, predict the trend found in the $1 / \tau_{50 \%}$ versus $T_{c}$ curves indicating that the theory can adequately fit the data. Figure $4 \mathrm{c}$ shows Avrami indexes between 2.5 to 4 , for all the tested $T_{c}$ values, indicating that the samples formed spherulites that grew instantaneously (for $n$ values between 2.5 and 3.4) or sporadically (for $n$ values between 3.5 and 4), depending on $T_{c}$. The obtained Avrami indexes reflect that the superstructures formed during the crystallization are independent of the even-odd effect.

Figure $4 \mathrm{~d}$ shows the enthalpy of crystallization $\left(\Delta H_{c}\right)$ values measured during the isothermal DSC runs at each $T_{c}$. Even though there is not a clear alternation between the $\Delta H_{c}$ values of even and odd samples, the $\Delta H_{c}$ values for $n_{C H 2}=6$ to 8 are 
lower than those for $n_{\mathrm{CH} 2}=9$ to 12 , indicating two separated regions, the even-odd and the saturation region. Similar behavior is also found during the non-isothermal tests (see Table S3 on the SI).

\subsubsection{Lauritzen and Hoffman (LH) Theory}

We have applied the LH theory (see Section S4 on SI) with the $T_{m}{ }^{\circ}$ values obtained by modifying the $T_{m \text {, end }}$ from SSA experiments (see Figure S4d), and the equilibrium melting enthalpies $\left(\Delta H_{m}{ }^{\circ}\right)$ predicted with the group contribution theory of Van Krevelen $^{34}$ (see Table S2). The experimental data of Figure 4a was fitted by the LH theory and plotted as a function of $T_{c}$ in Figure 5a (solid lines are the results of the fits), and as a function of the supercooling, defined as $\Delta T=T_{m}{ }^{\circ}-T_{c}$ (see Figure 5b). The LH fits were also applied to the experimental data of Figure 3a.

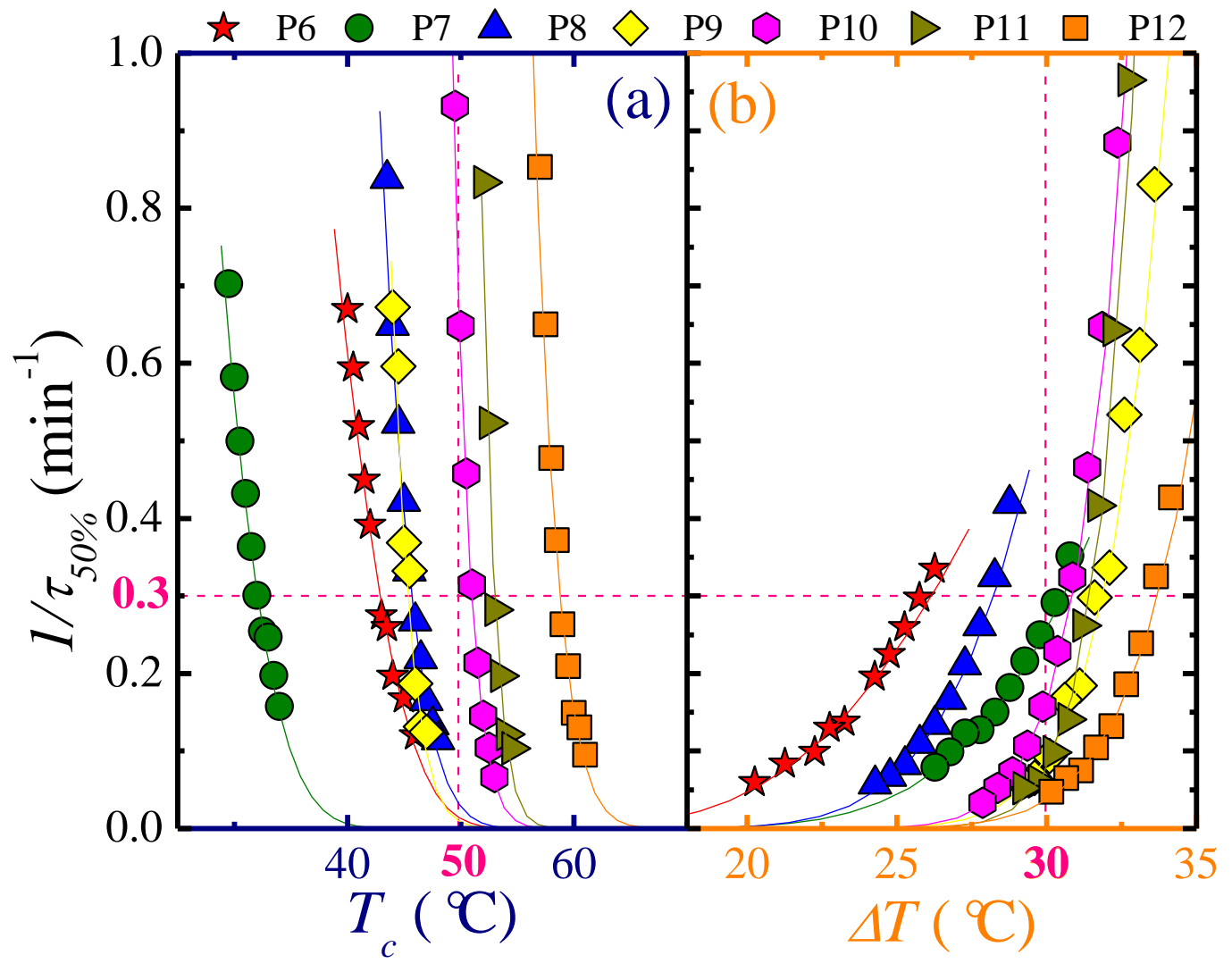


Figure 5. $1 / \tau_{50 \%}$ values as a function of (a) $T_{c}$ and (b) $\Delta T$. The solid lines represent the LH fit. Note that the horizontal and vertical dashed lines indicate the constant values selected for comparison purposes.

Figure 5 shows that the overall crystallization kinetics, in terms of both $T_{c}$ and supercooling can be separated into an even-odd region $\left(n_{\mathrm{CH} 2}=6\right.$ to 9$)$ and a saturated region $\left(n_{\mathrm{CH} 2}=10\right.$ to 12$)$. From the $1 / \tau_{50 \%}$ vs. $\Delta T$ curves, it can be observed that the samples in the even-odd and saturation region are brought together, confirming a certain thermodynamic influence in the kinetics. For comparison purposes we plot, in Figure 6 , the $T_{c}, \Delta T$ and $1 / \tau_{50 \%}$ as a function of $n_{C H 2}$, at constant $1 / \tau_{50 \%}=0.3 \mathrm{~min}^{-1}$ (Figures 6a and b), $T_{c}=50{ }^{\circ} \mathrm{C}$ (Figure 6c), and $\Delta T=30{ }^{\circ} \mathrm{C}$ (Figure 6d), respectively (note that the selected comparison values are indicated in Figure 5).

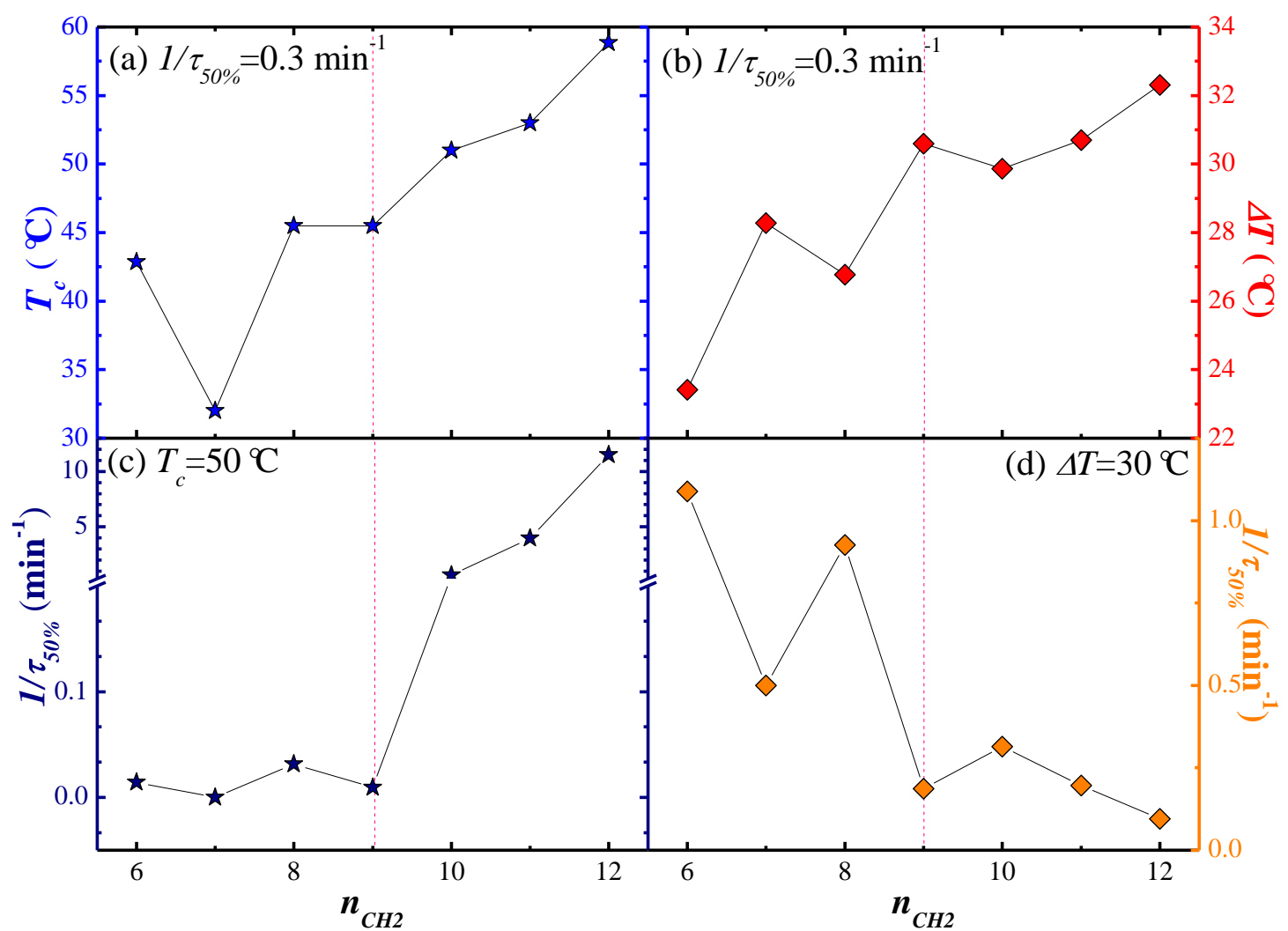

Figure 6. Comparison of (a) $T_{c}$ (at constant $1 / \tau_{50 \%}=0.3 \mathrm{~min}^{-1}$ ), (b) $\Delta T$ (at a constant $1 / \tau_{50 \%}=0.3 \min ^{-1}$ ), (c) $1 / \tau_{50 \%}$ (at a constant $T_{c}=50{ }^{\circ} \mathrm{C}$ ) and (d) $1 / \tau_{50 \%}$ (at a constant $\Delta T=30{ }^{\circ} \mathrm{C}$ ) as a function of $n_{\mathrm{CH} 2}$. 
Figure 6 shows that independently of the parameter taken as a constant, two regions are observable, the even-odd region $\left(n_{\mathrm{CH} 2}=6\right.$ to 9$)$ and the saturation region $\left(n_{C H 2}=10\right.$ to 12$)$. In some plots (see Figures $6 a$ and $\left.c\right)$, there is a slight alternation in the saturation region; this might indicate that the interaction between the functional groups is weak, but do not totally disappear upon increasing $n_{\mathrm{CH} 2}$. The LH parameters were calculated for all the samples, and the nucleation and growth contribution were estimated (see Tables S5 to S7, and Figure S5) according to the procedure described by Lorenzo and Müller. ${ }^{35}$ The assumptions made applying the LH theory were focused on the differences between the even-odd region and the saturation region. Significant differences were found between the even-odd and the saturation region in line with the other experimental results. Further details of these differences are discussed in Section S4 of the SI.

\section{3. $\quad$ SN and SSA}

We performed SN and SSA experiments for all the samples. All the samples show the three SN domains during the SN test, i.e., Domain I, II, and III. In the even-odd region, the samples showed a broader Domain II (memory effect) in comparison to the saturation region, in which the Domain II width remains constant (see Figure S7h). However, in general, Domain II width is reduced as $n_{C H 2}$ increases, corroborating the findings of Sangroniz et al. ${ }^{21}$ The crystalline memory can also be evaluated by examining the width of Domain IIa. In this work, only the PC6 and PC7 samples exhibit Domain IIa (see Figures S7a and b). The samples with $n_{\mathrm{CH} 2}>7$, do not present Domain IIa (see Figure S7c to g) because the strength of the interactions is reduced with the incorporation of methylene groups, as was found by Sangroniz et al. ${ }^{21}$ These 
results suggest that neither the width of Domain II nor that of Domain IIa depends on the even-odd effect.

We have performed SSA tests on all samples. For comparison purposes, the highest $T_{s \text {, ideal }}$ was first employed, which corresponded to that of the PC12, i.e., $T_{s}$, ideal $=68{ }^{\circ} \mathrm{C}$. The results are shown in Figure 7. We also performed SSA tests by taking the $T_{s, \text { ideal }}$ of each material, obtaining relatively similar SSA final heatings (see Figure S8).

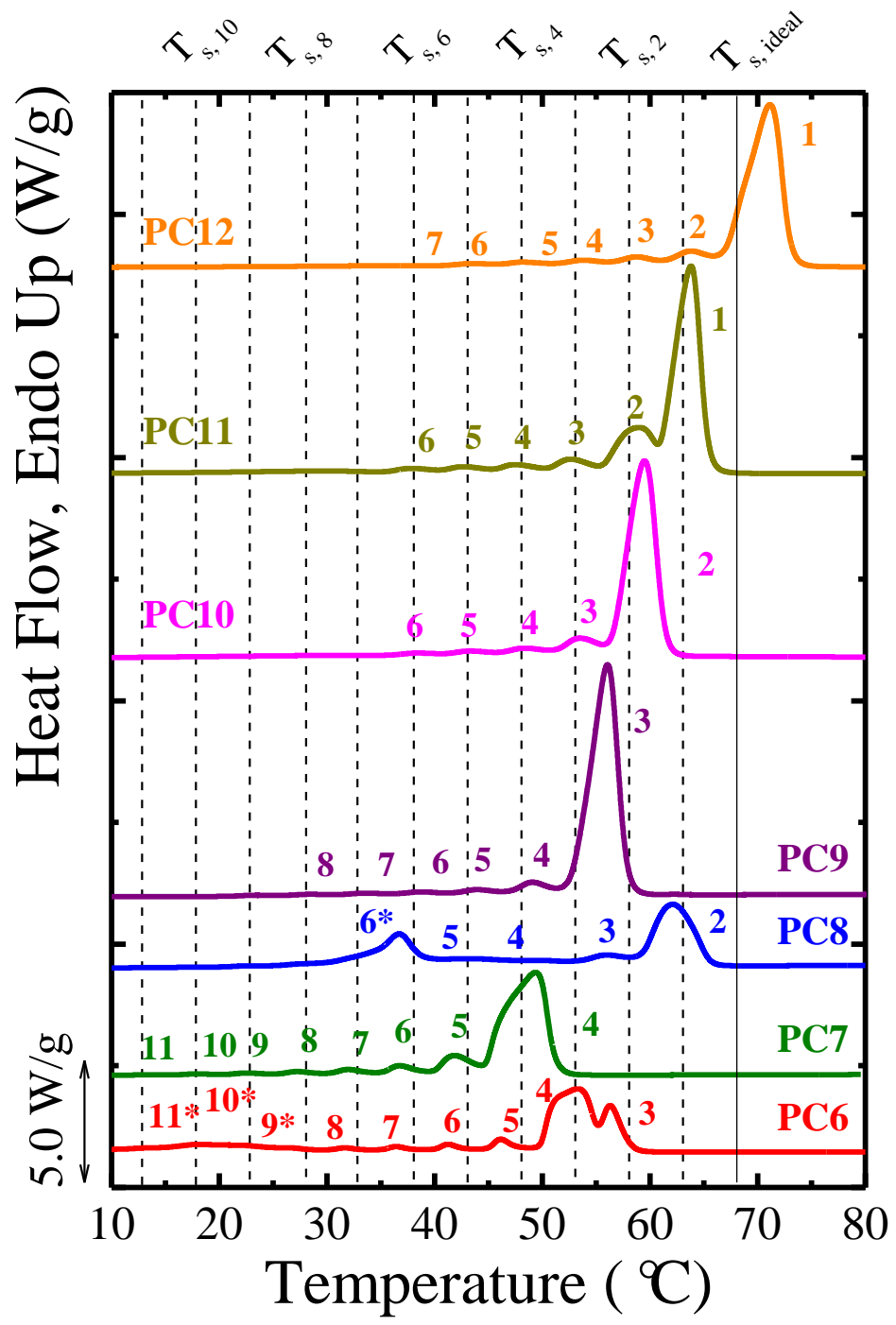

Figure 7. Final heating after the SSA protocol was applied to the indicated samples. We used the following conditions for the SSA protocol: $T_{s, \text { ideal }}=68{ }^{\circ} \mathrm{C}$, a fractionation windows of $5{ }^{\circ} \mathrm{C}$, and scan rates of $20{ }^{\circ} \mathrm{C} / \mathrm{min}$. 
Figure 7 shows that all the samples can be fractionated by SSA. In this case, the molecular fractionation provoked by the SSA protocol fractionates the materials based on their molecular weight distributions, as they are linear polymers with no defects that can interrupt their crystallizable units. The final DSC scan after the SSA protocol produces one or two high temperature abundant fractions (as judged by their enthalpy) and minor fractions at lower temperatures. The thermal fractions has been numbered in Figure 7. Each fraction represents the melting of a group of crystals with similar average lamellar thickness. ${ }^{28-31,40-42}$

If we consider the highest melting fraction of each material in Figure 7, we can see that the SSA protocol also generates two separated regions (i.e., even-odd and saturation region). Let us consider that fraction 1 is generated by $T_{s, 1}$; fraction 2 by $T_{s \text {, }}$ 2, and so on, as $T_{s, \text { ideal }}$ only provokes self-nucleation. ${ }^{28}$ In the even-odd region $\left(n_{\mathrm{CH} 2}=6\right.$ to 9), we can observe how there is an alternation of the melting points of the maximum melting point fractions. The even samples exhibit higher melting points than the odd samples. 


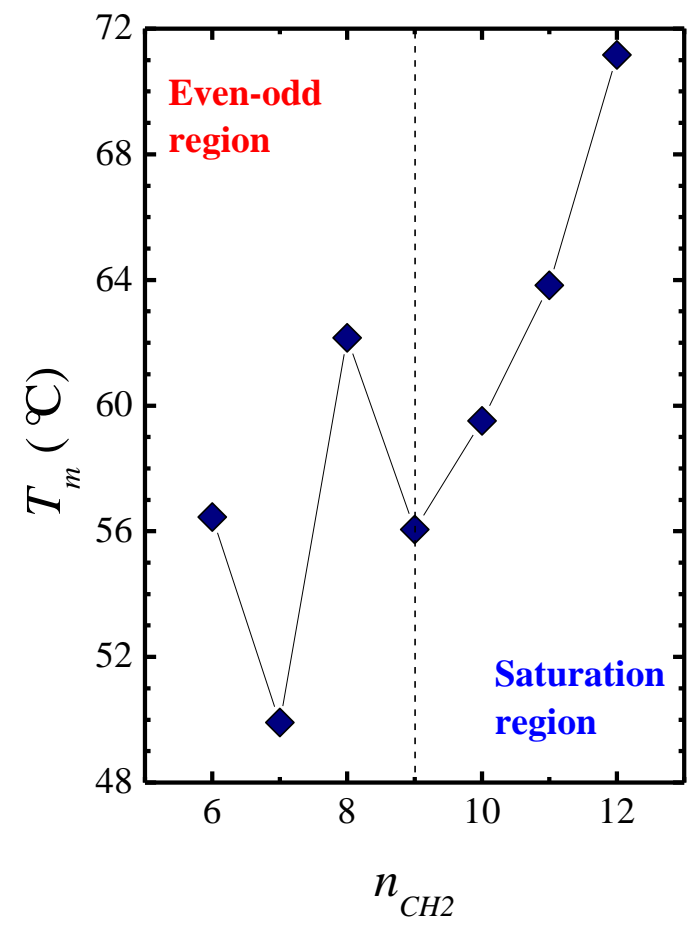

Figure 8. Highest $T_{m}$ value obtained after SSA of each sample (i.e., the $T_{m}$ of the highest melting point fraction) as a function of $n_{\mathrm{CH} 2}$.

In the saturation region, it is observed that $T_{m}$ of the higher melting point fraction increases as $n_{C H 2}$ increases. Figure 8 clearly shows the alternation of the highest $T_{m}$ values after SSA in the even-odd region. At higher $n_{C H 2}$ values, a monotonic increase in $T_{m}$ values is observed in the saturation region, demonstrating that these two regions are independent of the crystallization conditions (i.e., non-isothermal, isothermal or SSA).

It is worth noting that in Figure 7, besides the even-odd effect, the PC6 and PC8 SSA profiles shown some peculiar low temperature fractions labeled $10^{*}$ and $6^{*}$ respectively that could be related to solid-solid transitions. The possible solid-solid transitions are beyond the scope of the present paper and will be studied in a forthcoming paper. 


\subsection{X-rays Analysis}

Figure 9 shows the WAXS patterns taken at room temperature (RT) for all the samples (also WAXS patterns taken during the cooling and subsequent heating are shown in Figure S9). Two main crystalline reflections are observed in every sample. For $n_{C H 2}=6$ to 9 , there is a shift in the positions of the diffraction peaks, depending on the value of $n_{\mathrm{CH} 2}$, indicating different lattice structures. On the other hand, for $n_{\mathrm{CH} 2}$ $=10$ to 12 , the peak positions are practically the same, despite the increase of $n_{C H 2}$; therefore, it is assumed that these samples have the same crystalline structure. Such behavior is in line with the even-odd region and saturation region described in previous Sections.

As far as we are aware, the crystalline structure of aliphatic polycarbonates has only been reported for the poly (trimethylene carbonate) (PC3) by Takahashi and Kojima $^{43}$ and the PC8 by Zhao et al. ${ }^{13}$ Takahashi and Kojima ${ }^{43}$ found that all the reflections for the PC3 can be indexed to an orthorhombic unit cell with $a=0.702$ $\mathrm{nm}, b=0.581 \mathrm{~nm}$, and $c$ (fiber axis) $=1.230 \mathrm{~nm}$, with their main peaks at $(110)$ and $(200)$ planes, and two molecular chains in the unit cell. Zhao et al. ${ }^{13}$ found that the $\alpha$ and $\beta$ form of the PC8 are both monoclinic. The $\alpha$-form, which is the one obtained in this work, has unit cell parameters equal to: $a=0.77 \mathrm{~nm}, b=1.01 \mathrm{~nm}, c$ (chain axis) $=2.52$ $\mathrm{nm}$, and $\beta=31.5^{0}$, and their main peaks at $q=14.5\left(2 \theta=20.45^{\circ}\right)$ and $16.4 \mathrm{~nm}^{-1}$ $\left(2 \theta=23.14^{\circ}\right)($ i.e., $d$-spacing of 0.430 and $0.380 \mathrm{~nm}$, respectively) are indexed to the (110) and (200) planes, respectively. The $\beta$-form is only found upon heating, and more details can be found in reference 13 . 


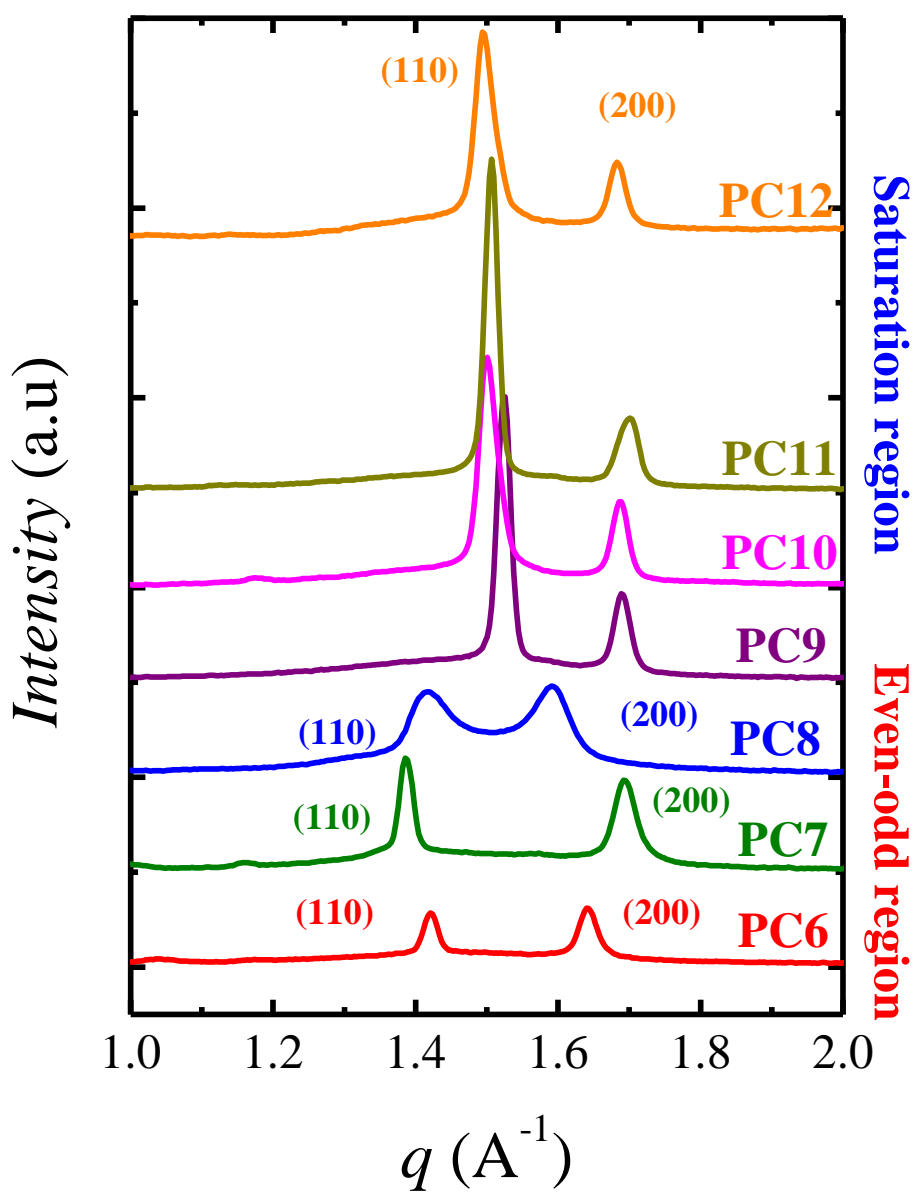

Figure 9. WAXS patterns for all the samples taken at RT. We indexed the main reflections of the samples to the (110) and (200) planes according to the lattice parameters reported by Masubuchi et al. ${ }^{44}$ in polycarbonate-glycols oligomers (i.e., $n_{\mathrm{CH} 2}=4$ to 10 ) and the known structures of $\mathrm{PC} 3^{43}$ and $\mathrm{PC} 8^{13}$ samples.

In the case of PCs with higher $n_{\mathrm{CH} 2}\left(\right.$ e.g., $n_{\mathrm{CH} 2}=10$ and 12), it has been considered that the unit cell resembles the orthorhombic unit cell of linear PE, with $a=0.740 \mathrm{~nm}, b=0.493 \mathrm{~nm}$, and $c$ (fiber axis) $=0.2534 \mathrm{~nm}$, and the very strong and strong reflections correspond to the (110) and (200) planes. ${ }^{45}$ For instance, Liu et al. ${ }^{46}$ assigned the reflections of the PC10 and PC12 samples to the orthorhombic PE unit cell, as well as Arandia et al. ${ }^{20}$ in the case of the PC12 sample. 
Masubuchi et al. ${ }^{44}$ studied by WAXS polycarbonate-glycols (PCg) oligomers with different $n_{\mathrm{CH} 2}$ (i.e., $n_{\mathrm{CH} 2}=3$ to 10 ), and simulated their structures. The PCg are not only similar to PCs chemically, but also structurally (i.e., the PCg show similar $q$ positions of the main peaks, and similar intensities), as we show in our WAXS patterns, see Figure 9. Hence, to further understand the crystalline structure in this work, we have used, as a first approximation, the PCg to index the main peaks and unit cells of the unknown unit cells for some of the samples reported in this work (i.e., $n_{\mathrm{CH} 2}=6,7,9,10,11$, and 12). We have considered the known PCs unit cell, as well as those reported for $\mathrm{PCg}$ oligomers. It is worth noting that more accurate crystallographic details of each unit cell would have to be performed using WAXS date on highly oriented samples, however, such determination is outside of the scope of the present work.

For the even-odd region, even samples $\left(n_{\mathrm{CH} 2}=6\right.$ and 8) were assigned to a monoclinic unit cell, with their main reflections corresponding to the (110) and (200) planes. In contrast, odd samples $\left(n_{\mathrm{CH} 2}=7\right.$ and 9$)$ were assigned to an orthorhombic unit cell with their main reflections corresponding to the (110) and (200) planes. For the saturation region, $n_{\mathrm{CH} 2}=10$ to 12 , the samples were assigned to an orthorhombic unit cell. Table 2 shows the $q$ values and calculated $d$-spacing of the main peaks. For comparison purposes, we have taken the unit cell parameters reported by Masubuchi et al. ${ }^{44}$ (see Section S7 of the Supporting information) and calculated the $d$-spacings. We found similarities between the $d$-spacings ( $d$-spacing ${ }^{1}$ vs. $d$-spacing $\left.{ }^{2}\right)$, indicating that the selected planes fit correctly with the reported unit cells. 
Table 2. $q$ and $2 \theta$ positions (i.e., taken from the WAXS patterns in Figure 9), and the calculated $d$-spacing, the assigned plane, and unit cell for all the samples, from $n_{\mathrm{CH} 2}=6$ to 12 .

\begin{tabular}{|c|c|c|c|c|c|c|}
\hline $\boldsymbol{n}_{\mathrm{CH} 2}$ & $\begin{array}{c}q \\
\left(\mathrm{~nm}^{-1}\right)\end{array}$ & $\begin{array}{c}2 \theta \\
\text { (degrees) }\end{array}$ & $\begin{array}{c}d \text {-spacing }{ }^{1} \\
(\mathrm{~nm})\end{array}$ & $\begin{array}{c}d \text {-spacing }^{2} \\
(\mathrm{~nm})\end{array}$ & $\begin{array}{c}\text { Assigned } \\
\text { plane }\end{array}$ & $\begin{array}{l}\text { Unit } \\
\text { cell }\end{array}$ \\
\hline \multirow[t]{2}{*}{6} & 14.2 & 20.1 & 0.441 & 0.435 & 110 & monoclinic \\
\hline & 16.8 & 23.8 & 0.373 & 0.378 & 200 & \\
\hline \multirow[t]{2}{*}{7} & 13.9 & 19.5 & 0.455 & 0.448 & 110 & orthorhombic \\
\hline & 17.2 & 24.4 & 0.364 & 0.366 & 200 & \\
\hline \multirow[t]{2}{*}{8} & 14.2 & 20.1 & 0.441 & 0.448 & 110 & monoclinic \\
\hline & 16.6 & 23.5 & 0.378 & 0.385 & 200 & \\
\hline \multirow[t]{2}{*}{9} & 15.4 & 21.8 & 0.411 & 0.413 & 110 & orthorhombic \\
\hline & 17.2 & 24.4 & 0.364 & 0.369 & 200 & \\
\hline \multirow[t]{2}{*}{10} & 15.1 & 21.3 & 0.417 & 0.414 & 110 & orthorhombic \\
\hline & 17.2 & 24.3 & 0.366 & 0.370 & 200 & \\
\hline \multirow[t]{2}{*}{11} & 15.1 & 21.3 & 0.417 & - & 110 & orthorhombic \\
\hline & 17.2 & 24.3 & 0.366 & - & 200 & \\
\hline \multirow[t]{2}{*}{12} & 15.1 & 21.3 & 0.417 & - & 110 & orthorhombic \\
\hline & 17.2 & 24.3 & 0.366 & - & 200 & \\
\hline
\end{tabular}

\footnotetext{
${ }^{1}$ Calculated $d$-spacing from WAXS data in Figure $10 .{ }^{2}$ Calculated $d$-spacing for unit cell parameters
} reported by Masubuchi et al. ${ }^{44}$ and assuming the main planes as (110) and (200).

Table 2 shows that in the even-odd region, the even samples $\left(n_{\mathrm{CH} 2}=6\right.$ and 8$)$ have similar positions, corresponding to the values reported in the literature for the $\mathrm{PC}^{3,33,44,47}$ and PC ${ }^{13,46}$ samples. As we mentioned before, by comparison, we ascribed the found reflections to the (110) and (200) planes of the PC8 monoclinic unit cell. In this work, the $\beta$-PC8 form is only found in the PC8 sample (at high 
temperature) upon heating (see the convergence of the two main peaks into a single one at high temperatures in Figure S9c on the SI).

According to Table 2, there is an alternation of structures in the even-odd region, monoclinic vs. orthorhombic. Such alternation was also found by Masubuchi et al., who reported a monoclinic unit cell for the even PCg's $\left(n_{C H 2}=4,6\right.$, and 8) and an orthorhombic unit cell, for odd PCg's $\left(n_{\mathrm{CH} 2}=5,7\right)$, with a slightly tilted angle (e.g., the $\alpha$ for the $n_{C H 2}=7$ is $85^{\circ}$ instead of $\left.90^{\circ}\right){ }^{44}$ It is worth noting that the PC3 (i.e., $n_{\mathrm{CH} 2}=3$ ) also has an orthorhombic unit cell. ${ }^{43}$ For monomeric units per cell, Masubuchi et al. ${ }^{44}$ reported 4 and 2 units for even and odd samples, respectively. This is in line with the reported units for the PC3 and PC8 samples, corroborating the similarities between PCs and PCg.

In the saturation region, $n_{\mathrm{CH} 2}=10$ to 12 , Table 2 shows that the samples have similar WAXS patterns that can be attributed to the weakening of the intramolecular interaction of the carbonate groups due to the longer methylene chain. Consequently, the WAXS patterns are comparable to those exhibited by linear PE, as well as PCg with $n_{\mathrm{CH} 2}=9$ and 10 .

Taking into account the assigned monoclinic unit cell for $n_{\mathrm{CH} 2}=6$, and 8 , and the orthorhombic unit cell for $n_{C H 2}=7,9$, and 10 to 12 , as well as their respective (110) and (200) planes, we have calculated the $d$-spacing by using the Bragg's Law (see Table 2) and the $a$ and $b$-axis sizes. These values are plotted as a function of $n_{C H 2}$ in Figure 10. For more details, see Table S9 on Section S8 of the Supporting Information. 

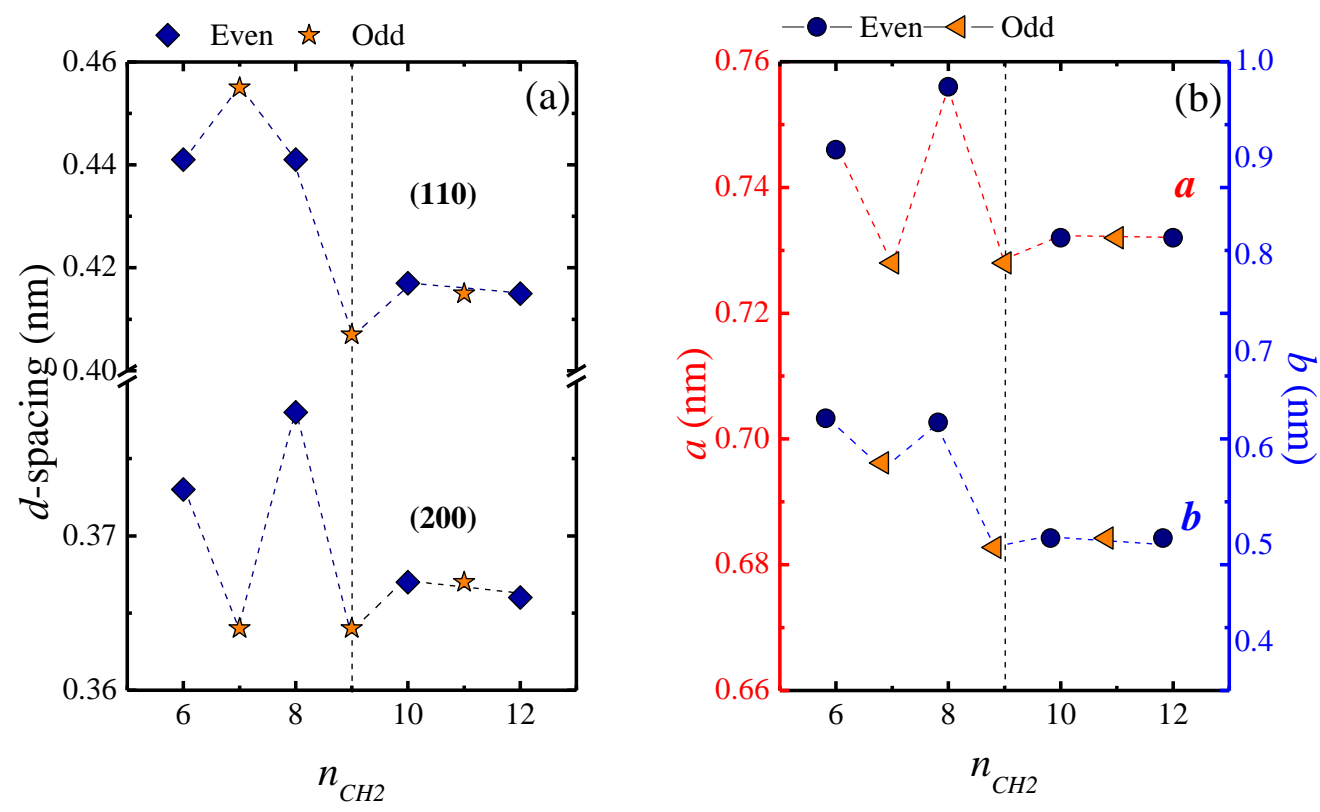

Figure 10. $d$-spacing as a function of $n_{C H 2}$. The $d$-spacings were calculated from the experimental data obtained in Figure $\mathrm{S} 9$ and Table $\mathrm{S} 9$ at $-40{ }^{\circ} \mathrm{C}$.

Figure 10a shows an alternation of the $d$-spacing (see Figure 10a) for the (110) and (200) planes, from $n_{C H 2}=6$ to 9 , due to the even-odd effect that generates different crystalline structure (i.e., even (monoclinic) vs. odd (orthorhombic)). The alternation is also observed in the $a$ and $b$-axis sizes (see Figure 10b), as is expected. Masubuchi et al. ${ }^{44}$ also found an alternation of the axis sizes in the PCg's. For $n_{\mathrm{CH} 2}=10$ to 12 , we found that the $d$-spacings and crystalline sizes remain unchanged due to a saturation of the even-odd effect. The saturation region is characterized by the decreasing influence of the carbonate groups and the increasing influence of the methylene units, instead. Thus, the methylene units determined the crystalline structure. In the next section, we are going to show the influence of the even-odd effect on the molecular conformation of the chains, with FT-IR experiments. 


\section{5. $\quad$ FT-IR analysis}

The FT-IR spectra show that all the samples possess typical strong bands related to the methylene and carbonate groups vibrations. In the case of the absorption bands of the methylene group, the samples show the characteristics bands due to asymmetric and symmetric $-\mathrm{CH}_{2}$ - stretching vibrations ${ }^{3}$ at 2940 and $2860 \mathrm{~cm}^{-1}$, respectively. For the carbonate group: The $\mathrm{C}=\mathrm{O}^{3,47}$ stretching and the $\mathrm{O}-\mathrm{C}-\mathrm{O}$ asymmetric stretching vibrations $^{3}$ are located from 1737 to $1729 \mathrm{~cm}^{-1}$ and 1245 to $1229 \mathrm{~cm}^{-1}$, respectively (see Figure S10). The differences between even and odd samples were found in the $\mathrm{CH}_{2}$ bending vibration, and the $\mathrm{CH}_{2}$-rocking vibration bands.

\section{$\mathrm{CH}_{2}$ bending vibration bands}

Figure 11 shows the FT-IR spectra of all the samples in the range of 1430 to 1570 $\mathrm{cm}^{-1}$ (i.e., $\mathrm{CH}_{2}$ bending region). In this range, we analyzed the differences in the conformational changes of the methylene group, on the basics of variation of the absorption bands assigned to a trans- or gauche-related conformation. ${ }^{48}$

For comparison purposes, the FT-IR spectra of all the samples were measured when the material is crystallized until saturation (i.e., we consider $T=T_{c, p e a k}-5{ }^{\circ} \mathrm{C}$. Note that $T$ is indicated in Figure 11). Figure 11 shows the bending vibration absorption bands corresponding to the methylene group, which can be categorized into two regions: the even-odd region characterized by bands related to the absorption of a trans or gauche-dominated conformation, and the saturation region that shows bands similar to linear PE crystallized in orthorhombic crystals. 


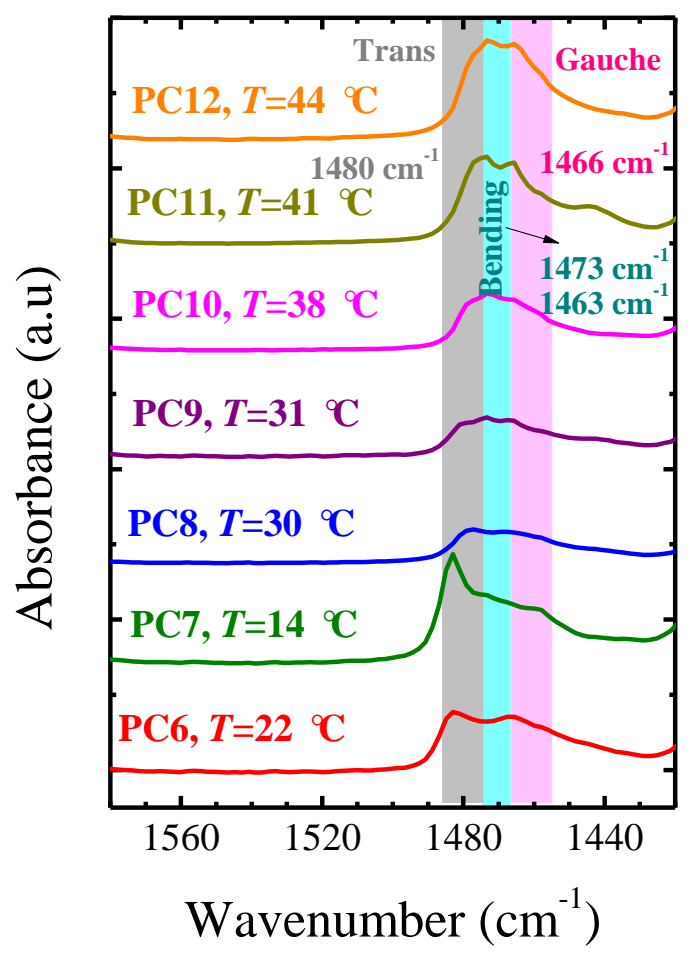

Figure 11. Comparison of all the samples at $5{ }^{\circ} \mathrm{C}$ below their $T_{c}$ (i.e., to ensure their saturated crystallization) in the $\mathrm{CH}_{2}$-bending bands. The shaded pink area indicates the gauche-related band, whereas the grey one indicates the trans-related band. The shaded blue space, in the middle, shows the PE-like bands.

In the even-odd region $\left(n_{\mathrm{CH} 2}=6\right.$ to 8$)$, we found that the even samples $\left(n_{\mathrm{CH} 2}=6\right.$, and 8) show the characteristic absorption of the methylene group with a mixture of gauche $\left(1466 \mathrm{~cm}^{-1}\right)$ and trans $\left(1481 \mathrm{~cm}^{-1}\right)$ conformations. The relative intensity of these two bands $\left(I_{1481} / I_{1466}\right)$, at the indicated temperatures in Figure 11, is slightly higher than 1. Such behavior was also found for PC8 by Zhao et al. ${ }^{13}$, in the $\alpha$-phase (i.e., trans-dominated), whereas the $\beta$-phase shows a trans/gauche coexistence (i.e., $\left.I_{1481} / I_{1466}<1.0\right)$, as we show in Figure $S 11$.

For PC7 $\left(n_{\mathrm{CH} 2}=7\right)$, the bending vibration absorption bands are shifted to 1475 and $1483 \mathrm{~cm}^{-1}$, respectively. Also, there is a shoulder peak located at $1460 \mathrm{~cm}^{-1}$, 
complicating the analysis of PC7. However, the encountered bands can also be attributed to the gauche and trans-dominated conformation. In this case, the dominant conformation is trans-dominated conformation, as is shown in Figure 11. In this case, the trans-related peak is more pronounced; therefore $I_{1483} / I_{1475}>1.0$. It is worth noting that in the even-odd region, the $\mathrm{CH}_{2}$ bending bands, for both even and odd samples, are shifted due to the attachment of the $\mathrm{CH}_{2}$ to a carbonate group.

In the saturation region, it is observed that the spectra of samples $\left(n_{\mathrm{CH} 2}=9-12\right)$ are similar, showing two bands, located at 1467 and $1473 \mathrm{~cm}^{-1}$. These bands are similar to those of $\mathrm{PE},{ }^{49}$ i.e., 1463 and $1473 \mathrm{~cm}^{-1}$ bands originated from the packaging of two polymer chains in an orthorhombic lattice. ${ }^{50}$ The shift of these bands, in comparison with PE bands, is related to the carbonyl groups. By comparing these bands with those of the even-odd region, their shift indicates the decreasing influence of the carbonyl groups. As the influence of the carbonyl group is diluted, the materials behave as PE, explaining the similarity in the conformation of the methylene groups (in the saturation region) with those adopted by PE chains crystallized in the orthorhombic crystal. Such dilution of the carbonyl group influence, was also found in the memory effect.

The above conformational bands analysis confirms the different crystalline structures between even and odd samples (see Figure 9). In the even-odd region, both even and odd samples have a trans-dominated conformation pronounced for the PC7 sample. In contrast, an important shift in the $\mathrm{CH}_{2}$-bending bands is observed in the saturation region, indicating the dilution of the carbonyl groups influence, and hence, the higher dominance of the interactions among methylene groups.

\section{$\mathrm{CH}_{2}$-rocking bands}


In Figure 12, we analyzed the absorption bands in the range of 780-700 $\mathrm{cm}^{-1}$, which correspond to a part of the progression band series (rocking-twisting mode) of $n$-paraffin with $n_{\mathrm{CH} 2}=6$ to $12 .^{51-53}$ In the saturation region, $n_{\mathrm{CH} 2}=10$ to 12 , two absorption bands can be observed, which are similar to the crystalline bands of the orthorhombic phase of PE, indicating the similarities between the PC10 to PC12 samples with the PE-type lattice. In the case of the odd samples, two similar bands appear due to their crystallization in an orthorhombic unit cell. In contrast, the even samples, which are crystallized in a monoclinic unit cell, only show a single peak.

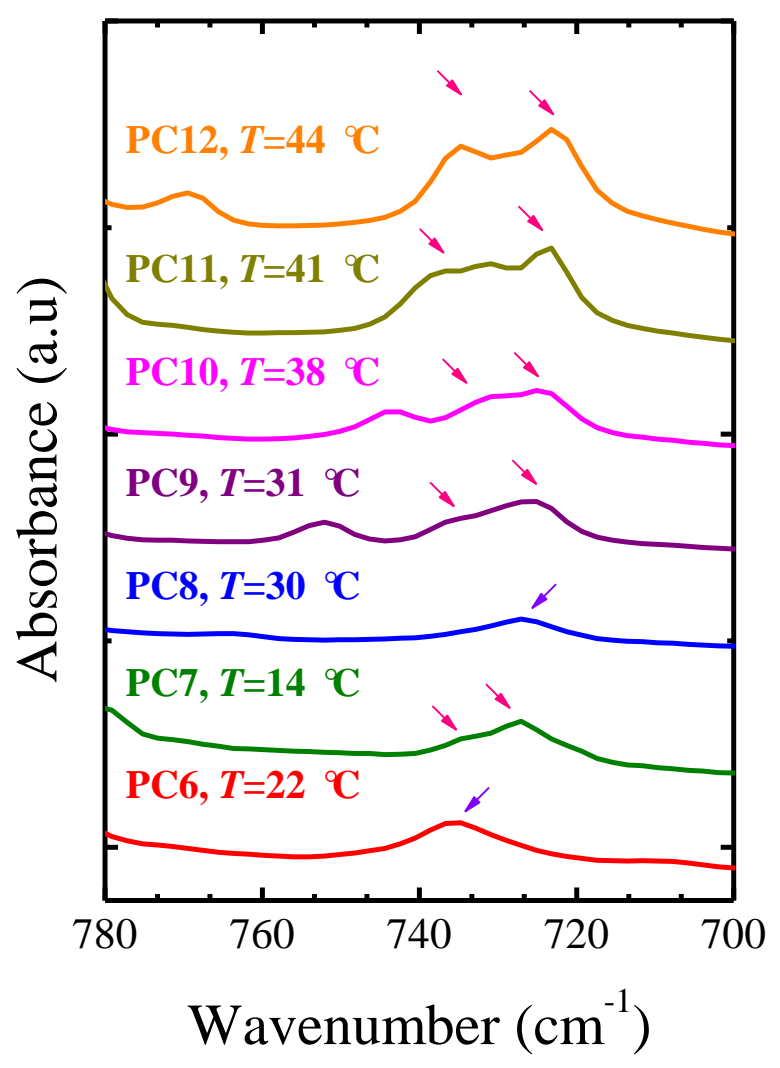

Figure 12. Comparison of all the samples at $5{ }^{\circ} \mathrm{C}$ below their $T_{c}$ (i.e., to ensure their total crystallization) in the $\mathrm{CH}_{2}$-rocking bands. The arrows indicate the main peak, and its splitting depending on the case. 
According to the above results, the intramolecular interactions influence the molecular conformations, hence their packaging and crystalline structure. Thus, both even and odd samples have a trans-dominated conformation, with $\mathrm{CH}_{2}$-bending bands shifted due to the influence of the carbonyl groups, and they crystallize in a monoclinic and orthorhombic unit cell, respectively. In contrast, in the saturation region, the interactions are dominated by the methylene groups, generating a molecular conformation dominated by the PE like structure and crystallization in an orthorhombic unit cell.

\section{Conclusions}

We characterized a series of aliphatic polycarbonates with different $n_{\mathrm{CH} 2}$ (6 to 12 ), synthesized by organocatalysis. The explored crystallization conditions were nonisothermal, modified and unmodified isothermal, and Successive Self-nucleation and annealing (SSA) experiments. Independently of the crystallization conditions, all the samples show a strong even-odd effect in thermal properties, for $n_{C H 2}=6$ to 9 , and saturation of the even-odd effect for $n_{C H 2}=10$ to 12 .

The even-odd effect region was characterized by the higher thermal properties, such as $T_{c}, T_{m}, 1 / \tau_{50 \%}^{G}, 1 / \tau_{50 \%}$, and higher final $T_{m}$ values obtained by SSA, of the even samples in comparison with the odd ones, describing an alternation or zigzag behavior as $n_{C H 2}$ increases. Such differences are attributed to the different crystalline structures, monoclinic (even) vs. orthorhombic (odd), and different intramolecular interactions generated by the carbonate groups reflected in the position of the trans and gauche related bands, and the higher prevalence of trans-dominated conformation in the odd sample. The influence of the carbonate groups is diluted as $n_{\mathrm{CH} 2}$ increases, as is 
reflected in the decrease of the memory effect in the even-odd region. However, the memory effect becomes weaker as $n_{\mathrm{CH} 2}$ increases, independently of the even or odd nature of the sample.

In contrast, in the saturation of the even-odd effect region, the properties increase as $n_{\mathrm{CH} 2}$ increases, indicating the dominance of the methylene groups interactions (over the carbonate groups). Such dominance generates the same crystalline structure (i.e., orthorhombic), conformation, and memory effect, independently of the increase in $n_{\mathrm{CH} 2}$.

\section{ACKNOWLEDGMENTS}

This work is supported by the National Key R\&D Program of China (2017YFE0117800) and the National Natural Science Foundation of China $(51820105005,21922308$, and 52050410327). We would like to thank the financial support provided by the BIODEST project; this project has received funding from the European Union's Horizon 2020 research and innovation programme under the Marie Skłodowska-Curie grant agreement No 778092. This work has also received funding from MINECO through project MAT2017-83014-C2-1-P and from the Basque Government through grant IT1309-19. R.A.P-C is supported by PIFI of the Chinese Academy of Science for international postdoctoral researchers (2019PE0004), and the China Postdoctoral Science Foundation (2020M670462). G.L. is grateful to the Youth Innovation Promotion Association of the Chinese Academy of Sciences (Y201908). L.M thanks Spanish Ministry of Education, Culture, and Sport for the predoctoral FPU fellowship received to carry out this work. 
Supporting Information. Protocols of the isothermal, non-isothermal, SN and SSA tests, degree of crystallinity, Lauritzen-Hoffman theory and analysis, Avrami theory and analysis, SN and SSA results, calculated d-spacings from PC-glycols, WAXS temperature-dependent experiments and unit cell parameters calculations, and additional FTIR results.

\section{References}

1. Su, W.; Feng, J.; Wang, H.-F.; Zhang, X.-Z.; Zhuo, R.-X. Controllable preparation of poly(alkylene carbonate)s and observation on their structure-related odd-even effect. Polymer 2010, 51 (5), 1010-1015 DOI: https://doi.org/10.1016/j.polymer.2010.01.009.

2. Sisti, L.; Totaro, G.; Marchese, P., PBS Makes its Entrance into the Family of Biobased Plastics. In Biodegradable and Biobased Polymers for Environmental and Biomedical Applications, Kalia, S., Avérous, L., Eds. 2016; pp 225-285.

3. Zhu, W.; Huang, X.; Li, C.; Xiao, Y.; Zhang, D.; Guan, G. High-molecularweight aliphatic polycarbonates by melt polycondensation of dimethyl carbonate and aliphatic diols: synthesis and characterization. Polymer International 2011, 60 (7), 1060-1067 DOI: 10.1002/pi.3043.

4. García-Martín, M. G.; Pérez, R. R.; Hernández, E. B.; Espartero, J. L.; MuñozGuerra, S.; Galbis, J. A. Carbohydrate-Based Polycarbonates. Synthesis, Structure, and Biodegradation Studies. Macromolecules 2005, 38 (21), 8664-8670 DOI: 10.1021/ma050676k.

5. Rokicki, G. Aliphatic cyclic carbonates and spiroorthocarbonates as monomers. Progress in Polymer Science 2000, 25 (2), 259-342 DOI: https://doi.org/10.1016/S0079-6700(00)00006-X.

6. Artham, T.; Doble, M. Biodegradation of Aliphatic and Aromatic Polycarbonates. Macromolecular Bioscience 2008, 8 (1), 14-24 DOI: 10.1002/mabi.200700106.

7. Pranamuda, H.; Chollakup, R.; Tokiwa, Y. Degradation of Polycarbonate by a Polyester-Degrading Strain, <em>Amycolatopsis $</ \mathrm{em}>\mathrm{sp}$. Strain HT-6. Applied and Environmental Microbiology 1999, 65 (9), 4220-4222.

8. Zhang, Z.; Kuijer, R.; Bulstra, S. K.; Grijpma, D. W.; Feijen, J. The in vivo and in vitro degradation behavior of poly(trimethylene carbonate). Biomaterials 2006, 27 (9), 1741-1748 DOI: https://doi.org/10.1016/j.biomaterials.2005.09.017.

9. Zhu, K. J.; Hendren, R. W.; Jensen, K.; Pitt, C. G. Synthesis, properties, and biodegradation of poly(1,3-trimethylene carbonate). Macromolecules 1991, 24 (8), 1736-1740 DOI: 10.1021/ma00008a008.

10. van Sliedregt, A.; Knook, M.; Hesseling, S. C.; Koerten, H. K.; de Groot, K.; van Blitterswijk, C. A. Cellular reaction on the intraperitoneal injection of four types of polylactide particulates. Biomaterials 1992, 13 (12), 819-824 DOI: https://doi.org/10.1016/0142-9612(92)90174-M. 
11. Rücker, M.; Laschke, M. W.; Junker, D.; Carvalho, C.; Schramm, A.; Mülhaupt, R.; Gellrich, N.-C.; Menger, M. D. Angiogenic and inflammatory response to biodegradable scaffolds in dorsal skinfold chambers of mice. Biomaterials 2006, 27 (29), 5027-5038 DOI: https://doi.org/10.1016/j.biomaterials.2006.05.033.

12. Meabe, L.; Lago, N.; Rubatat, L.; Li, C.; Müller, A. J.; Sardon, H.; Armand, M.; Mecerreyes, D. Polycondensation as a Versatile Synthetic Route to Aliphatic Polycarbonates for Solid Polymer Electrolytes. Electrochimica Acta 2017, 237, 259266 DOI: https://doi.org/10.1016/j.electacta.2017.03.217.

13. Zhao, T.-P.; Ren, X.-K.; Zhu, W.-X.; Liang, Y.-R.; Li, C.-C.; Men, Y.-F.; Liu, C.-Y.; Chen, E.-Q. "Brill Transition" Shown by Green Material Poly(octamethylene carbonate). ACS Macro Letters 2015, 4 (3), 317-321 DOI: 10.1021/acsmacrolett.5b00045.

14. Zhang, J.; Zhu, W.; Li, C.; Zhang, D.; Xiao, Y.; Guan, G.; Zheng, L. Effect of the biobased linear long-chain monomer on crystallization and biodegradation behaviors of poly(butylene carbonate)-based copolycarbonates. RSC Advances 2015, 5 (3), 2213-2222 DOI: 10.1039/c4ra10466h.

15. Park, J. H.; Jeon, J. Y.; Lee, J. J.; Jang, Y.; Varghese, J. K.; Lee, B. Y. Preparation of High-Molecular-Weight Aliphatic Polycarbonates by Condensation Polymerization of Diols and Dimethyl Carbonate. Macromolecules 2013, 46 (9), 3301-3308 DOI: 10.1021/ma400360w.

16. Bossion, A.; Heifferon, K. V.; Meabe, L.; Zivic, N.; Taton, D.; Hedrick, J. L.; Long, T. E.; Sardon, H. Opportunities for organocatalysis in polymer synthesis via step-growth methods. Progress in Polymer Science 2019, 90, 164-210 DOI: https://doi.org/10.1016/j.progpolymsci.2018.11.003.

17. Mutlu, H.; Ruiz, J.; Solleder, S. C.; Meier, M. A. R. TBD catalysis with dimethyl carbonate: a fruitful and sustainable alliance. Green Chemistry 2012, 14 (6), 1728-1735 DOI: 10.1039/c2gc35191a.

18. Sun, J.; Kuckling, D. Synthesis of high-molecular-weight aliphatic polycarbonates by organo-catalysis. Polymer Chemistry 2016, 7 (8), 1642-1649 DOI: 10.1039/c5py01843a.

19. Nederberg, F.; Connor, E. F.; Möller, M.; Glauser, T.; Hedrick, J. L. New Paradigms for Organic Catalysts: The First Organocatalytic Living Polymerization. Angewandte Chemie International Edition 2001, 40 (14), 2712-2715 DOI: 10.1002/1521-3773(20010716)40:14<2712::aid-anie2712>3.0.co;2-z.

20. Arandia, I.; Meabe, L.; Aranburu, N.; Sardon, H.; Mecerreyes, D.; Müller, A. J. Influence of Chemical Structures on Isodimorphic Behavior of Three Different Copolycarbonate Random Copolymer Series. Macromolecules 2020, DOI: 10.1021/acs.macromol.9b02078.

21. Sangroniz, L.; Sangroniz, A.; Meabe, L.; Basterretxea, A.; Sardon, H.; Cavallo, D.; Müller, A. J. Chemical Structure Drives Memory Effects in the Crystallization of Homopolymers. Macromolecules 2020, 53 (12), 4874-4881 DOI: 10.1021/acs.macromol.0c00751.

22. Thalladi, V. R.; Boese, R.; Weiss, H.-C. The Melting Point Alternation in $\alpha, \omega-$ Alkanedithiols. Journal of the American Chemical Society 2000, 122 (6), 1186-1190 DOI: $10.1021 /$ ja9934221.

23. Costa, J. C. S.; Santos, L. M. N. B. F. Chain-Length Dependence of the Thermodynamic Behavior of Homologous $\alpha, \omega$-Disubstituted Alkanes. Journal of Chemical \& Engineering Data 2019, 64 (6), 2229-2246 DOI: 10.1021/acs.jced.9b00125. 
24. Soccio, M.; Lotti, N.; Finelli, L.; Gazzano, M.; Munari, A. Aliphatic poly(propylene dicarboxylate)s: Effect of chain length on thermal properties and crystallization kinetics. Polymer 2007, 48 (11), 3125-3136 DOI: https://doi.org/10.1016/j.polymer.2007.04.007.

25. Papageorgiou, G. Z.; Bikiaris, D. N. Crystallization and melting behavior of three biodegradable poly(alkylene succinates). A comparative study. Polymer 2005, 46 (26), 12081-12092 DOI: https://doi.org/10.1016/j.polymer.2005.10.073.

26. Kazuhiko, S.; Yun, C.; Kazutoshi, F.; Noriyuki, Y.; Masaki, H. Odd-Even Effect in the Optical Resolution by Optically Active Polyamides Having (-)-Anti Head-to-head Coumarin Dimer Component. Chemistry Letters 1988, 17 (4), 647-650 DOI: $10.1246 / \mathrm{cl} .1988 .647$.

27. Lorenzo, A. T.; Arnal, M. L.; Albuerne, J.; Müller, A. J. DSC isothermal polymer crystallization kinetics measurements and the use of the Avrami equation to fit the data: Guidelines to avoid common problems. Polymer Testing 2007, 26 (2), 222-231 DOI: https://doi.org/10.1016/j.polymertesting.2006.10.005.

28. Müller, A. J.; Michell, R. M.; Pérez, R. A.; Lorenzo, A. T. Successive Selfnucleation and Annealing (SSA): Correct design of thermal protocol and applications. European Polymer Journal 2015, 65, 132-154 DOI: https://doi.org/10.1016/j.eurpolymj.2015.01.015.

29. Müller, A. J.; Lorenzo, A. T.; Arnal, M. L. Recent Advances and Applications of "Successive Self-Nucleation and Annealing" (SSA) High Speed Thermal Fractionation. Macromolecular Symposia 2009, 277 (1), 207-214 DOI: 10.1002/masy.200950325.

30. Müller, A. J.; Hernández, Z. H.; Arnal, M. L.; Sánchez, J. J. Successive selfnucleation/annealing (SSA): A novel technique to study molecular segregation during crystallization. Polymer Bulletin 1997, 39 (4), 465-472 DOI: 10.1007/s002890050174 31. Müller, A. J.; Arnal, M. L. Thermal fractionation of polymers. Progress in Polymer Science 2005, 30 (5), 559-603 DOI: https://doi.org/10.1016/j.progpolymsci.2005.03.001.

32. Fernández, C. E.; Bermudez, M.; Versteegen, R. M.; Meijer, E. W.; Muller, A. J.; Muñoz-Guerra, S. Crystallization studies on linear aliphatic n-polyurethanes. Journal of Polymer Science Part B: Polymer Physics 2009, 47 (14), 1368-1380 DOI: 10.1002/polb.21736.

33. Berti, C.; Celli, A.; Marchese, P.; Marianucci, E.; Marega, C.; Causin, V.; Marigo, A. Aliphatic poly(alkylene dithiocarbonate)s: Thermal properties and structural characteristics of poly(hexamethylene dithiocarbonate). Polymer 2007, 48 (1), 174-182 DOI: https://doi.org/10.1016/j.polymer.2006.11.035.

34. Van Krevelen, D. W., CHAPTER 5 - CALORIMETRIC PROPERTIES. In Properties of Polymers (Third Edition), Van Krevelen, D. W., Ed. Elsevier: Amsterdam, 1997; pp 109-127.

35. Lorenzo, A. T.; Müller, A. J. Estimation of the nucleation and crystal growth contributions to the overall crystallization energy barrier. Journal of Polymer Science Part B: Polymer Physics 2008, 46 (14), 1478-1487 DOI: 10.1002/polb.21483.

36. Gedde, U. W., Polymer Physics. Springer Netherlands: 1999; p 298.

37. Avrami, M. Granulation, Phase Change, and Microstructure Kinetics of Phase Change. III. The Journal of Chemical Physics 1941, 9 (2), 177-184 DOI: $10.1063 / 1.1750872$.

38. Lauritzen, J. I.; Hoffman, J. D. Theory of Formation of Polymer Crystals with Folded Chains in Dilute Solution. J. Res. Natl. Bur. Stand., Sect. A 1960, 64A (1), 73- 
102 DOI: http://dx.doi.org/10.6028/jres.064A.007.

39. Hoffman, J. D.; Lauritzen, J. I. Crystallization of Bulk Polymers With Chain Folding: Theory of Growth of Lamellar Spherulites J. Res. Natl. Bur. Stand., Sect. A 1961, 65A (4), 297-336 DOI: http://dx.doi.org/10.6028/jres.065A.035.

40. Lorenzo, A. T.; Arnal, M. L.; Müller, A. J.; Lin, M.-C.; Chen, H.-L. SAXS/DSC Analysis of the Lamellar Thickness Distribution on a SSA Thermally Fractionated Model Polyethylene. Macromolecular Chemistry and Physics 2011, 212 (18), 2009-2016 DOI: 10.1002/macp.201100240.

41. Lorenzo, A. T.; Arnal, M. L.; Müller, A. J.; Boschetti de Fierro, A.; Abetz, V. High Speed SSA Thermal Fractionation and Limitations to the Determination of Lamellar Sizes and Their Distributions. Macromolecular Chemistry and Physics 2006, 207 (1), 39-49 DOI: 10.1002/macp.200500437.

42. Arandia, I.; Mugica, A.; Zubitur, M.; Iturrospe, A.; Arbe, A.; Liu, G.; Wang, D.; Mincheva, R.; Dubois, P.; Müller, A. J. Application of SSA thermal fractionation and X-ray diffraction to elucidate comonomer inclusion or exclusion from the crystalline phases in poly(butylene succinate-ran-butylene azelate) random copolymers. Journal of Polymer Science Part B: Polymer Physics 2016, 54 (22), 2346-2358 DOI: 10.1002/polb.24146.

43. Takahashi, Y.; Kojima, R. Crystal Structure of Poly(trimethylene carbonate). Macromolecules 2003, 36 (14), 5139-5143 DOI: 10.1021/ma030076q.

44. Masubuchi, T.; Sakai, M.; Kojio, K.; Furukawa, M.; Aoyagi, T. Structure and Properties of Aliphatic Poly(carbonate) glycols with Different Methylene Unit Length. e-Journal of Soft Materials 2007, 3, 55-63 DOI: 10.2324/ejsm.3.55.

45. Clark, E. S., Unit Cell Information on Some Important Polymers. In Physical Properties of Polymers Handbook, Mark, J. E., Ed. Springer New York: New York, NY, 2007; pp 619-624.

46. Liu, X.; Pang, C.; Ma, J.; Gao, H. Random Copolycarbonates Based on a Renewable Bicyclic Diol Derived from Citric Acid. Macromolecules 2017, 50 (20), 7949-7958 DOI: 10.1021/acs.macromol.7b01641.

47. Kricheldorf, H. R.; Mahler, A. Polymers of carbonic acid 18: polymerizations of cyclobis(hexamethylene carbonate) by means of $\mathrm{BuSnCl} 3$ or $\mathrm{Sn}$ (II)2ethylhexanoate. Polymer 1996, 37 (19), 4383-4388 DOI: https://doi.org/10.1016/0032-3861(96)00288-1.

48. Zheng, R.-Q.; Chen, E.-Q.; Cheng, S. Z. D.; Xie, F.; Yan, D.; He, T.; Percec, V.; Chu, P.; Ungar, G. Phase Identification in a Series of Liquid Crystalline TPP Polyethers and Copolyethers Having Highly Ordered Mesophase Structures. 8. Phase and Structural Evolution in a Series of Copolyethers Containing Odd-Numbered Methylene Units in Both Comonomers. Macromolecules 2000, 33 (14), 5159-5168 DOI: $10.1021 / \mathrm{ma} 991963 \mathrm{p}$.

49. Gulmine, J. V.; Janissek, P. R.; Heise, H. M.; Akcelrud, L. Polyethylene characterization by FTIR. Polymer Testing 2002, 21 (5), 557-563 DOI: https://doi.org/10.1016/S0142-9418(01)00124-6.

50. Zerbi, G.; Gallino, G.; Del Fanti, N.; Baini, L. Structural depth profiling in polyethylene films by multiple internal reflection infra-red spectroscopy. Polymer 1989, 30 (12), 2324-2327 DOI: https://doi.org/10.1016/0032-3861(89)90269-3.

51. Snyder, R. G.; Schachtschneider, J. H. Vibrational analysis of the $\mathrm{n}$ paraffins-I: Assignments of infrared bands in the spectra of C3H8 through nC19H40. Spectrochimica Acta 1963, 19 (1), 85-116 DOI: https://doi.org/10.1016/0371-1951(63)80095-8. 
52. Schachtschneider, J. H.; Snyder, R. G. Vibrational analysis of the $\mathrm{n}$ paraffins-II: Normal co-ordinate calculations. Spectrochimica Acta 1963, 19 (1), 117-168 DOI: https://doi.org/10.1016/0371-1951(63)80096-X.

53. Kobayashi, S.; Tadokoro, H.; Chatani, Y. Structural studies on polyethers, [(CH2)m-O-]n. VI. The higher members with $\mathrm{m}=6-10,12$. Die Makromolekulare Chemie 1968, 112 (1), 225-241 DOI: 10.1002/macp.1968.021120120. 